\title{
Geometrical envelopes: extending graphical contemporary niche theory to communities and eco-evolutionary dynamics
}

\author{
Thomas Koffel ${ }^{\mathrm{a}, \mathrm{b}, *}$, Tanguy Daufresne ${ }^{\mathrm{a}}$, François Massol ${ }^{\mathrm{c}}$, Christopher A. \\ Klausmeier $^{\mathrm{b}}$ \\ ${ }^{a}$ UMR EcoßSSols, Campus Supagro, 2 place Viala, 34060 Montpellier, France \\ ${ }^{b}$ Kellogg Biological Station (Michigan State University), 3700 E Gull Lake Dr, Hickory \\ Corners, MI 49060, United States \\ ${ }^{c} C N R S$, Université de Lille - Sciences et Technologies, UMR 8198 Evo-Eco-Paleo, F-59655 \\ Villeneuve d'Ascq, France
}

\begin{abstract}
Contemporary niche theory is a powerful structuring framework in theoretical ecology. First developed in the context of resource competition, it has been extended to encompass other types of regulating factors such as shared predators, parasites or inhibitors. A central component of contemporary niche theory is a graphical approach popularized by Tilman that illustrates the different outcomes of competition along environmental gradients, like coexistence and competitive exclusion. These food web modules have been used to address species sorting in community ecology, as well as adaptation and coexistence on eco-evolutionary time scales in adaptive dynamics. Yet, the associated graphical approach has been underused so far in the evolutionary context. In this paper, we provide a rigorous approach to extend this graphical method to a continuum of interacting strategies, using the geometrical concept of the envelope. Not only does this approach provide community and eco-evolutionary bifurcation diagrams along environmental gradients, it also sheds light on the similarities and differences
\end{abstract}

\footnotetext{
*Corresponding author

Email addresses: thomas.koffel@supagro.inra.fr (Thomas Koffel), tanguy.daufresne@inra.fr (Tanguy Daufresne), francois.massol@univ-lille1.fr (François Massol), klausme1@msu.edu (Christopher A. Klausmeier)

Preprint submitted to Journal of Theoretical Biology

July 10, 2016
}

(C) 2016. This manuscript version is made available under the Elsevier user license http://www.elsevier.com/open-access/userlicense/1.0/ 
between those two perspectives. Adaptive dynamics naturally merges with this ecological framework, with a close correspondence between the classification of singular strategies and the geometrical properties of the envelope. Finally, this approach provides an integrative tool to study adaptation between levels of organization, from the individual to the ecosystem.

Keywords: Coexistence, competition, zero net growth isocline, community ecology, species sorting, adaptive dynamics, evolutionary branching

\section{Introduction}

Competition is a ubiquitous interaction among living organisms, and thus a major driver of community structure and evolution by natural selection. As such, it was at the core of the very first mathematical models of population 5 dynamics and theoretical ecology (Lotka, 1925, Volterra, 1926, Gause, 1934). However, the explicit inclusion of resources for which species compete only came several decades later with the pioneering works of MacArthur and colleagues (MacArthur and Levins, 1964, MacArthur and Wilson, 1967, MacArthur, 1970). Mechanistic competition models, or modules, allow a useful graphical representation introduced by MacArthur and Levins (1964), developed by León and Tumpson (1975) and largely popularized by Tilman (1980, 1982), which summarizes graphically the different outcomes of competition along environmental gradients, delimiting the coexistence regions from competitive exclusion or founder control. This method relies on the combination of three key graphical ingredients: Zero Net Growth Isoclines (ZNGIs), consumption/impact vectors and supply points. These respectively represent a species' minimal requirements, its consumptive impacts, and the externally-driven resource availability in the absence of any consumer. Supported empirically (Miller et al., 2005), the theory also brings strong conceptual results on the conditions for coexistence 
consistent with the competitive exclusion principle (Levin, 1970).

The concept of resource competition can be generalized to encompass any kind of regulating factors that mediate interspecific interactions. This was done by Chase and Leibold (2003) under the unifying umbrella of "contemporary niche theory", further formalized by recent developments (Meszéna et al., 2006, Barabás et al., 2014b). For example, two prey sharing one predator formally behave as if they were competing for a single resource, a situation referred to as apparent competition by Holt (1977). This allows the use of the graphical representation in this generalized framework, as was done with apparent competition plus resource competition by Holt et al. (1994), Grover (1995), Leibold (1996) and Chase and Leibold (2003). Interference competition through explicit inhibitory product emission also fits in this framework, with the inhibitor playing the role of a regulating factor (Gerla et al., 2009). Recently, several authors further extended this graphical approach to take into account several phenomena: nutrient cycling (Daufresne and Hedin, 2005), cooperation (de Mazancourt and Schwartz, 2010), niche construction (Kylafis and Loreau, 2011) and population structure, either spatial (Ryabov and Blasius, 2011, Haegeman and Loreau, 2015) or demographic (Loreau and Ebenhöh, 1994, Schellekens et al., 2010).

Popularized in the context of a couple of interacting species, these niche theoretic models can be scaled up to investigate community assembly, based on the idea that local environmental conditions are the drivers of species sorting from a large or potentially infinite number of species along a trade-off curve (Leibold et al., 2004). This approach relies on the key assumption that 'everything is 45 everywhere', namely that there is no dispersal limitation to a species' presence (Baas Becking, 1934, De Wit and Bouvier, 2006). The associated generalized 
graphical methods uses the concept of geometrical envelopes, the boundary of a family of curves, providing a natural way to look graphically at species sorting along an environmental gradient (Armstrong, 1979, Tilman, 1980, 1982, Lei-

so bold, 1996, Chase and Leibold, 2003, Schade et al., 2005, Danger et al., 2008). Community composition and levels of regulating factors along the gradient can thus be investigated. These mechanistic models naturally fit into trait-based approaches, which have garnered recent interest in ecology (Lavorel and Garnier, 2002, McGill et al., 2006, Westoby and Wright, 2006, Litchman and Klaus55 meier, 2008). Traits hold the key to linking trade-offs from the organism level to ecosystem functions and services, in both aquatic and terrestrial ecosystems (Litchman et al., 2007, Lavorel and Grigulis, 2012). Trait-based approaches also are a natural framework to study community responses to climate change (Adler et al., 2012, Thomas et al., 2012, Barabás et al., 2014b).

60

Simultaneously, it has long been recognized that organisms are the product of their evolutionary history (Dobzhansky, 1973) and there is growing evidence of the interplay between ecology and evolution (Thompson, 1998, Yoshida et al., 2003, Hairston et al., 2005, Grant and Grant, 2006, Stuart et al., 2014).

${ }_{65}$ However, the influence of this past or present evolution on food web modules remains understudied. Theoretically, those questions have been addressed during the last decades using Adaptive Dynamics (Hofbauer and Sigmund, 1990, Geritz et al., 1997, 1998, Dieckmann and Law, 1996). This powerful framework allows one to address evolution in arbitrarily complex ecological models. As an 7o evolutionary game theory approach, this is done by including the density- and frequency-dependent selection arising from the feedback loop between the evolving population and its environment (Dieckmann and Metz, 2006). It clarifies the conditions under which evolution acts as an optimizing process (Dieckmann 
and Ferrière, 2004, Metz et al., 2008) and leads to the concept of evolutionary branching, a potential prelude to diversification (Geritz et al., 1997, 1998, Metz et al., 1996, Dieckmann and Doebeli, 1999). Unlike species sorting, adaptive dynamics considers local invasibility only. Evolution can thus get stuck on local but not global fitness maxima. When applied to food web modules, this enables one to investigate the evolutionary stability of coexistence in various ecological situations (Schreiber and Tobiason, 2003, Shoresh et al., 2008, Klausmeier et al., 2007, Zu et al., 2015). Yet the conditions that allow evolutionarily stable coexistence remain unclear, as ecological coexistence often vanishes on evolutionary time scales through convergent selection. Importantly, the graphical representation is still helpful in those adaptive competition modules to perform invasion analysis when combined with ZNGI geometrical envelopes (Meszéna and Metz, 1999).

The concept of the envelope has a long history in mathematical optimization and its applications. It has for example its own theorem in economics, the Envelope Theorem (Samuelson, 1947), and is related to the Pareto frontier (Pareto, 1906), a multi-objective optimization concept first introduced in economics and now commonly used in engineer and environmental sciences (Marler and Arora, 2004, Seppelt et al., 2013, Lester et al., 2013). Envelopes of environment-dependent growth rate functions have been used in ecology to identify the optimal species corresponding to given environmental conditions (Eppley, 1972, Norberg, 2013). In resource competition theory, the idea of taking the ZNGI envelope of a continuum of competing strategies can be traced back to Tilman (1982), who applied it heuristically to species sorting from a regional pool or adaptive foraging at the individual scale. It has been used more recently in the context of communities under the names 'community ZNGI' 
(Danger et al., 2008, Schade et al., 2005) or 'overall ZNGI' (Chase and Leibold, 2003). Meszéna and Metz (1999) introduced the ZNGI envelope in the ecoevolutionary context and called it 'the boundary'. They showed how evolution through the trait substitution process of adaptive dynamics can be pictured by ZNGIs rolling along their envelope, and how this helps identify evolutionary singularities and deduce their properties, both graphically.

The aim of the paper is to unite the theoretical approaches to community assembly processes and eco-evolutionary dynamics under the common umbrella of a graphical theory of interaction, using geometrical envelopes. This provides a promising tool to investigate adaptation, diversification and functioning along environmental gradients. We first review step-by-step how to apply the graphical method to competition modules with a few species, combining the concepts of invasion analysis and impact map. Then, we show through a rigorous mathematical framework how those ideas can be naturally extended to a continuum of competitors using geometrical envelopes. Building on the intuitions of Meszéna and Metz (1999), we demonstrate for general non-linear ZNGIs how their envelope geometry relates to local invasibility. Moreover, the use of the impact ray map allows us to identify and characterize geometrically the eco-evolutionary singularities associated with a given supply point. This provides both community and eco-evolutionary bifurcation diagrams, predicting a vast range of possible adaptive behaviors along the environmental gradients. Conditions leading to robust coexistence, evolutionary priority effects and branching points can be easily identified, as they present unambiguous graphical signatures. Conceptually, this graphical approach shows how adaptive dynamics naturally combines with mechanistic competition theory. It also emphasizes the similarities and differences between species sorting from a regional pool and evolution through 
small step mutations, a global versus local picture. The envelope approach provides a unified tool to navigate between scales through adaptation, from the example of a versatile model of competition on two resources (Schreiber and Tobiason, 2003), shedding new light on the conditions leading to the evolution of resource specialization.

\section{Modeling framework and analysis}

\subsection{Standard graphical construction for $n$ competitors}

Let us first introduce the general class of mathematical models treated in this paper. We consider a community of $n$ species, the abundances of which are denoted $\boldsymbol{N}=\left(N_{1}, N_{2}, \ldots N_{n}\right)$, which interact through $p$ regulating factors $\boldsymbol{R}=\left(R_{1}, R_{2}, \ldots R_{p}\right)$. The dynamics of $N_{j}$ and $R_{i}$ obey the following equations:

$$
\begin{aligned}
\frac{d N_{j}}{d t} & =w_{j}\left(R_{1}, R_{2}, \ldots, R_{p}\right) N_{j} \\
\frac{d R_{i}}{d t} & =l_{i}\left(S_{i}-R_{i}\right)+\sum_{j=1}^{n} I_{i j}\left(R_{1}, R_{2}, \ldots, R_{p}\right) N_{j}
\end{aligned}
$$

140

where $w_{j}$ is the net growth rate of population $j$ and $I_{i j}$ its per capita impact on the regulating factor $i$. No assumptions are made about their expression, except that they both only depend on the regulating factors $\boldsymbol{R}$. We thus follow Meszéna et al. (2006) by considering that interactions between individuals are indirect, only mediated by the regulating factors. As a particular case of Meszéna et al. 45 (2006), eq. (1b) assumes that the total impact of a population on a regulating factor is simply proportional to its density. Note that we have chosen the convention that resource consumption corresponds to negative impact $I_{i j}$ but also allowed positive $I_{i j}$, for example with shared predators. Finally, the supply point $\boldsymbol{S}=\left(S_{1}, S_{2}, \ldots S_{p}\right)$ and the leaching or mortality rates $l_{i}$ parametrize the semi-chemostat intrinsic dynamics of the regulating factors, which interact only indirectly, through the species $\boldsymbol{N}$. Particularly suited for experimental 
setups (Novick and Szilard, 1950, Monod, 1950), this framework is classically used to describe abiotic resource dynamics in a wide range of systems (Tilman, 1982, Grover, 1997, Loreau, 1998a). For biotic resources, a well chosen change 155 of variables can be made to map logistic growth into the chemostat dynamics of eq. (1b) (see Appendix A). Examples of models following the particular form of eq. (1) include resource-consumer modules (Tilman, 1980, Wolkowicz and Lu, 1992, Schreiber and Tobiason, 2003), food webs with keystone predation (Holt et al., 1994, Leibold, 1996), material-cycle models (Loreau, 1998b, 160 Daufresne and Hedin, 2005) and interference competition through inhibitory product emissions (Gerla et al., 2009).

We will restrict our analysis of this system to its equilibria. Setting aside unrealistic fine-tuning between the demographic parameters, this implies that the 165 maximal number of coexisting populations can not be greater than the number $p$ of regulating factors, a classical result known as the Competitive Exclusion Principle (CEP) or Dimension-Diversity Theorem (Levin, 1970, Gyllenberg and Meszéna, 2005, Meszéna et al., 2006). Because of the non-linear feedback loops between the regulating factors and the population densities, it is generally not 170 possible to find analytical expressions for the equilibria of eq. (1), except for some particular systems. However, it is possible to visualize those solutions graphically for up to three regulating factors, following a long tradition in theoretical ecology (León and Tumpson, 1975, Tilman, 1980, 1982, Leibold, 1996, Grover, 1997, Chase and Leibold, 2003). The aim here is to review this graph175 ical construction in the case of the general model of eq (1). Doing so, we will introduce the associated basic concepts and notations needed for our generalization of this approach to the community and evolutionary frameworks in the next sections. 
Table 1: Notation

\begin{tabular}{ccl}
\hline Notation & & Definition \\
\hline$i$ & & Regulating factor \\
$p$ & & index \\
$R_{i}$ & & total number \\
$S_{i}$ & & ensity \\
$l_{i}$ & & intrinsic per capita loss rate \\
\hline Finite case & Continuous case & Interacting population \\
$j$ & $x$ & index/trait value \\
$n$ & $\mathcal{X}$ & total number/trait space \\
$N_{j}$ & $N(x)$ & abundance \\
$w_{j}$ & $w(x)$ & per capita net growth rate \\
$I_{i j}$ & $I_{i}(x)$ & impact on regulating factor $i$ \\
$\tilde{I}_{i j}$ & $\tilde{I}_{i}(x)$ & renormalized impact on regulating factor $i$ \\
& $H(x)$ & second derivative of the invasion fitness \\
& $J(x)$ & derivative of the fitness gradient \\
\hline$\alpha$ & & Schreiber and Tobiason's model \\
$m$ & & resource interaction shape parameter \\
$g_{i}$ & $g(x)$ & per capita mortality rate \\
& & per capita gross growth rate \\
ZNGI & & Acronyms \\
CEP & & Zero Net Growth Isocline \\
ESS & & Competitive Exclusion Principle \\
CSS & & Evolutionarily Stable Strategy \\
PIP & & Convergence Stable Strategy \\
& & Pairwise Invasibility Plot \\
& &
\end{tabular}


The method consists of two steps: invasion analysis and impact map. This decomposes the environmental feedback loop into its sensitivity and impact components following the terminology of Meszéna and colleagues (Meszéna and Metz, 1999, Meszéna et al., 2006), which shares strong similarities with Chase and Leibold's (2003) concepts of requirement and impact niches. To help visualize our method, we will illustrate it by presenting the case of $n$ populations $N_{1}, N_{2}, \ldots N_{n}$ interacting through two regulating factors $R_{1}$ and $R_{2}$. While described in general terms in the main text, we will use a flexible resource competition model introduced by Schreiber and Tobiason (2003) as a concrete example (see Box 1 and Figures). The graphical construction of the ecological bifurcation diagram follows two distinct steps, that will naturally be extended later in the eco-evolutionary case.

Invasion analysis. Let us first focus our attention on the population equations, eq. (1a). At equilibrium, they imply for every population that either $N_{i}=0$ (the population is absent) or $w_{i}=0$ (its net growth rate is zero). There are thus three possible kind of equilibria: 'empty' or washout state with no population, one non-zero population only, or coexistence of two distinct populations with extinction of the other $n-2$ populations. Coexistence of three or more different populations on two regulating factors is not possible due to the CEP. From equation (1a), the presence of a population $i$ in the system restricts $R_{1}$ and $R_{2}$ to $w_{i}\left(R_{1}, R_{2}\right)=0$. Graphically, this defines the so-called Zero Net Growth Isocline of population $i$ or $\mathrm{ZNGI}_{i}$ (Tilman, 1980). For each competitor $i$, this curve delimits in the $\left(R_{1}, R_{2}\right)$ plane the regions where net growth is positive $\left(w_{i}>0\right)$ from the ones where net growth is negative $\left(w_{i}<0\right)$ (see Fig. 1). A direct implication is that coexistence between populations at equilibrium is 205 only possible for $\left(R_{1}, R_{2}\right)$ where their two ZNGIs intersect. On the contrary, the 
'empty' equilibrium with no population exists for any set of regulating factors, as none of the populations is imposing its zero net growth constraint on the regulating factors.

More importantly, the ZNGIs also allow one to investigate the stability against invasion of those equilibria through invasion analysis. A necessary condition for any equilibrium involving either 0,1 or 2 populations to be stable is for the point $\left(R_{1}, R_{2}\right)$ to be located outside the positive growth regions of all the other potential invaders (Tilman, 1982, Leibold, 1996). Thus, the potentially stable 'empty' equilibria are the ones for which $\left(R_{1}, R_{2}\right)$ are simultaneously located outside (in the direction of lowered growth rate) the whole set of ZNGIs (shaded regions in Fig. 1). Population $i$ stable equilibria can only be located on the portion of $\mathrm{ZNGI}_{i}$ that is outside all the other ZNGIs, and the same rule extends to stable coexistence points between two populations. The set of points $\left(R_{1}, R_{2}\right)$ corresponding to non-empty potentially stable equilibria form what we can call the outer envelope of the ZNGIs (thicker border in Fig. 1). To conclude, a ZNGI directly represents both the minimum requirements and the competitive ability of its corresponding population.

Supply point mapping. We have so far identified the candidate $\left(R_{1}, R_{2}\right)$ values associated with the different kinds of possible equilibria using eq. (1a) only. Yet the solutions $\left(R_{1}, R_{2}\right)$ also have to satisfy the limiting factor equations eq. (1b) set equal to zero. When solved together, this obviously makes them functions of the parameters of the system, including the supply point. To draw a bifurcation diagram, we would like to know for every supply point which are the corresponding $\left(R_{1}, R_{2}\right)$ at equilibrium and thus deduce its associated characteristics ('empty', one population or coexistence). In practice, the map is 
A

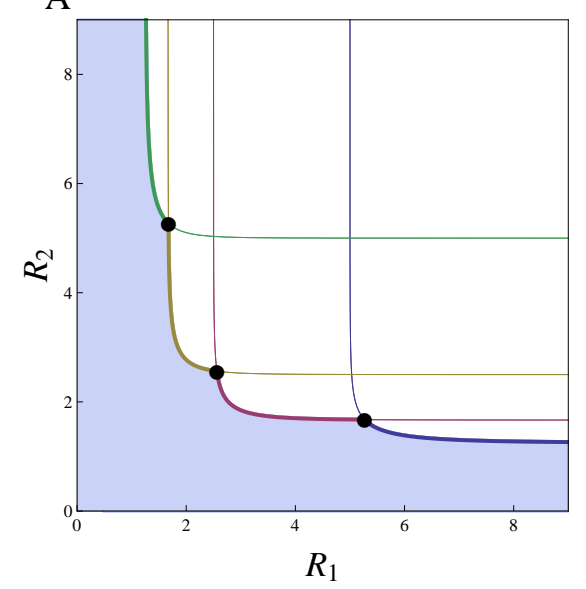

B

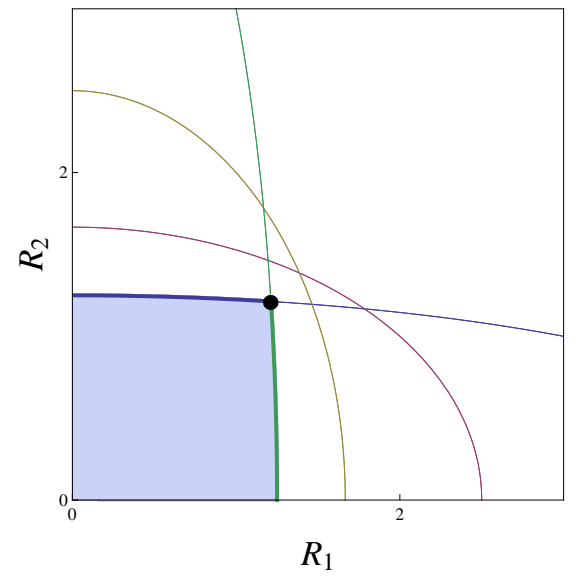

Figure 1: Invasion analysis for a set of 4 competing populations consuming two resources $R_{1}$ and $R_{2}$. Competitors are labeled with $i$ from 1 to 4 (respectively blue, purple, yellow, and green). They follow Schreiber and Tobiason (2003) model described in Box 1 with $x_{i}=0.2 i$ and $m=1$. Resources are either A) interactive essential $(\alpha=-5)$ or B) antagonistic $(\alpha=2)$. Invasion analysis consists in drawing the set of ZNGIs (thin), selecting its outer envelope corresponding to non-invadable single population equilibria (thick) and potential coexistence points (black dots); the stable 'empty' equilibria are located under the envelope (light blue). In the essential case, each strategy has a range of non-invadable equilibria and can potentially coexist with the neighboring populations. In the antagonistic case, the extreme strategies $\left(x_{1}=0.2\right.$ and $\left.x_{4}=0.8\right)$ exclude all the other ones but can potentially coexist together.

performed using the supplementary constraints emerging from equations (1b) taken at equilibrium. First, note that this map is trivial for the regulating factors associated with the 'empty' equilibrium. Indeed, having all the $N_{i}$ equal to 0 leads to $\boldsymbol{S}=\boldsymbol{R}$. This means that if we now draw the ZNGI envelope in the supply plane, all the supply points situated outside of it will map to the 'empty' equilibrium (White region, Fig. 2).

Where are the supply points leading to non-zero populations located? Let us first put aside the coexistence case, and focus on a single population equilibrium with $N_{i} \neq 0$ and $N_{j}=0$ for $i \neq j$. Setting eq. (1b) equal to zero leads to

$$
\boldsymbol{S}=\boldsymbol{R}+N_{i} \tilde{\boldsymbol{I}}_{\boldsymbol{i}}(\boldsymbol{R})
$$


where $\tilde{\boldsymbol{I}}_{\boldsymbol{i}}=\left(I_{1 i} / l_{1}, I_{2 i} / l_{2}\right)$ is the impact vector renormalized to account for dif-

245 of the envelope. Eq. (2) means that for a given regulating factor point on $\mathrm{ZNGI}_{i}$, the corresponding supply points $\boldsymbol{S}$ are located along the ray that starts from the point $\left(R_{1}, R_{2}\right)$ when $N_{i}=0$ and moves away from it following the direction vector $\tilde{\boldsymbol{I}}_{\boldsymbol{i}}$ as $N_{i}$ increases. This means that all the supply points along

In the coexistence case, the method is slightly different. Equations (1b) now leads to $\boldsymbol{S}=\boldsymbol{R}+N_{i} \tilde{\boldsymbol{I}}_{\boldsymbol{i}}(\boldsymbol{R})+N_{j} \tilde{\boldsymbol{I}}_{\boldsymbol{j}}(\boldsymbol{R})$ with $\boldsymbol{R}$ at the two ZNGIs' intersection. Thus, all the supply points situated in the cone originating at $\boldsymbol{R}$ and delineated by vectors $\tilde{\boldsymbol{I}}_{\boldsymbol{i}}$ and $\tilde{\boldsymbol{I}}_{\boldsymbol{j}}$ map to this coexistence equilibrium when both densities 265 not to be degenerate. However, this coexistence point is dynamically stable only if (see Appendix B):

$$
\left(I_{1 i} I_{2 j}-I_{1 j} I_{2 i}\right)\left(\frac{\partial w_{i}}{\partial R_{1}} \frac{\partial w_{j}}{\partial R_{2}}-\frac{\partial w_{i}}{\partial R_{2}} \frac{\partial w_{j}}{\partial R_{1}}\right)>0
$$

This is known as the mutual invasibility criterion and can be interpreted graph- 
A

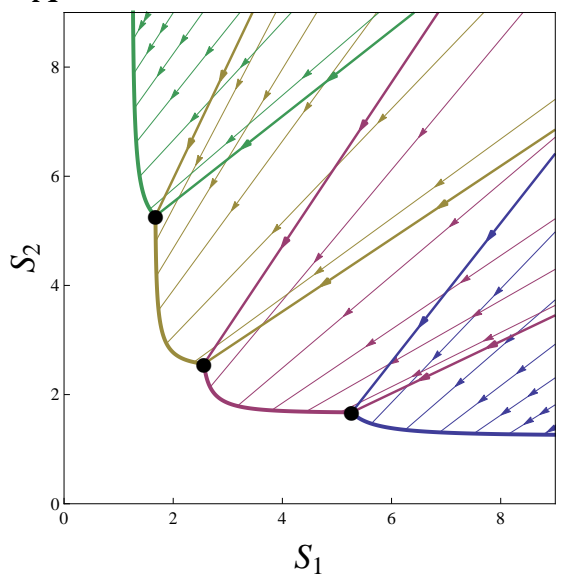

B

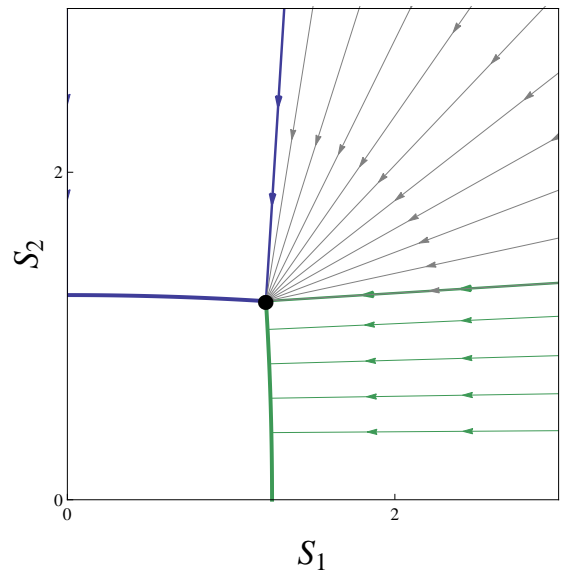

Figure 2: Supply point map for a set of 4 competiting populations consuming two resources $R_{1}$ and $R_{2}$ following the model of Box 1 . Same configuration and parameters as Fig. 1 . The stable ZNGI portions identified in Fig. 1 have been directly transposed into the supply point space. Starting from each non-invadable envelope portion (thick, plain), drawing a subset of the corresponding impact rays (thin, arrowed) helps visualizing the map that associates to every supply point its corresponding regulating factors at equilibrium. The boundaries of each portion map (thick, arrowed) combined with its corresponding ZNGI portion delimit zones of similar equilibria of the bifurcation diagram. A: there are four single-population zones (green, yellow, purple and blue) plus an empty one (white). Note that some of the former overlap, accounting for priority effects. B: there are two single-population zones (blue, green) plus an empty one (white) and a coexistence one (gray). Note that all the impact rays map to the same point in the latter case.

To summarize, an ecological bifurcation diagram as a function of the supply points $\left(S_{1}, S_{2}\right)$ can be obtained by combining ZNGIs and impact rays through the following steps: (1) Draw the ZNGIs of the different populations and identify their outer envelope. (2) Locate the regulating factor points corresponding to the 'empty' solutions (outside the envelope), population $i$ only solutions (on 
$\mathrm{ZNGI}_{i}$ portions of the envelope) and the coexistence solutions (where the latter portions intersect, typically making a 'kink' in the envelope). (3) Identify the regions of the supply point plane scanned by each population impact rays when 285 its origin moves along its corresponding ZNGI portion (a subset of impact rays can also be represented). (4) Identify potentially stable coexistence 'cones' from the kinks of the envelope.

There are two major advantages of this graphical approach. First, it delimits regions along the supply gradients for which a given species assemblage is present in the system. Historically, this allowed one to identify conditions for coexistence of two consumers (Tilman, 1982) and describe species succession along a nutrient gradient (Tilman, 1982, Leibold, 1996). Secondly, drawing the impact rays also allows one to deduce the equilibrium regulating factor levels and sometimes also population densities directly on the diagram. For consumers growing on essential resources, this is a way to identify which factor will be limiting for a given supply (Tilman, 1982). The direction of the impact vectors also enables one to assess the relative impact of a population on the regulating factors. But using impact rays has other advantages, which have been underused so far. Sometimes, a given supply point can be reached by several impact rays, which means that it maps to several distinct equilibria. In this case, the system presents alternative stable states, which is the basis of the 'founder effect'. There is an interesting diversity of situations that can be encountered depending on the states involved in this bistability. The most well-known case is one single population versus another one (Fig. 2A). But it is also possible to have alternative stable states inside a single population as it is often the case for structured populations (Schreiber and Rudolf, 2008, Guill, 2009, Schellekens et al., 2010), or between a community and an 'empty' state as it is common in 
the presence of a positive feedback loop or an ecosystem engineer (Scheffer et al., 2001, Rietkerk et al., 2004, Kéfi et al., 2010). The graphical method presented here allows one to identify alternative stable states regions in the bifurcation diagram without ambiguity.

With this graphical construction, we have provided only some necessary conditions for the local stability of the different equilibria through invasion analysis, but those are not always sufficient. For example, a single population may have alternative stable states through an Allee effect. These stable states are generally separated by unstable states that would not be identified explicitly as such by the scheme presented above. However, a careful study of the envelope of the impact rays moving along the ZNGI for a single-population equilibrium can supplement this by further restricting an impact ray to its stable portion (see Appendix B). This idea will be used again in the eco-evolutionary case to identify branching points (see supply point mapping in section 2.3). Limit cycles and other nonequilibrium attractors are also a possibility, even when criterion 325 (3) is satisfied, as the other stability criteria can not always be satisfied. Again, this would not be detectable on the graphical analysis. However, these two situations do not happen for simple systems like the standard two consumers on two resources (Tilman, 1982, Chase and Leibold, 2003, Schreiber and Tobiason, 2003), although they can for three or more resources (Huisman and Weissing,

\subsection{Extension to a continuum of competitors: the ZNGI envelope}

We now extend the previous framework from a discrete set of populations to a continuous set of strategies. The evolution of quantitative traits and phenotypic plasticity can be considered as occuring among a continuous set of strategies, as 335 can community assembly (Tilman, 1982, 1988, Chase and Leibold, 2003). Our 
motivation here is twofold. First, we aim at providing a rigorous framework to address the question of species sorting in communities and integrate this effect at the ecosystem scale. Second, this introduces the basic tools necessary to perform the eco-evolutionary analysis of the next section. This will highlight the similarities and differences between the species sorting and the adaptive dynamics approaches.

Formally, the generalization to a continuum of competitors is straightforward with the results of the previous section in mind. Omitting time-dependencies to lighten the notation, the dynamics of the system now reads:

$$
\begin{aligned}
\frac{\partial N(\boldsymbol{x})}{\partial t} & =w(\boldsymbol{R}, \boldsymbol{x}) N(\boldsymbol{x}) \\
\frac{d R_{i}}{d t} & =l_{i}\left(S_{i}-R_{i}\right)+\int_{\boldsymbol{x} \in \mathcal{X}} I_{i}(\boldsymbol{R}, \boldsymbol{x}) N(\boldsymbol{x})
\end{aligned}
$$

where the subscript $j$ is now replaced by its continuous analog, the trait vector $\boldsymbol{x}$, which contains the functional traits that fully describe the strategy of the population with density $N(\boldsymbol{x})$. The global impact of the competitors on regulating factor $i$ is now obtained by integrating the impact $I_{i}(\boldsymbol{R}, \boldsymbol{x})$ of every strategy $\boldsymbol{x}$ over the whole trait space $\mathcal{X}$. This trait space has to be seen as the collection of all the variable trait combinations that could possibly be present in the system. We assume in practice that $\mathcal{X}$ is a connected subset of a real vector space. Usually, this trait space is constrained by trade-offs inequalities which account for correlations between the traits. This usually excludes 'Hutchinsonian demons' that outcompete all other species (Kneitel and Chase, 2004). In practice, those trade-offs are often taken to be saturated, i.e. as equalities instead of inequalities, in order to reduce the dimensionality of the trait space. When not, they simply add boundaries to the trait space. Note that we work at equilibrium, so the CEP still applies, which means that the $N(\boldsymbol{x})$ can only 

ber of regulating factors $p$. From now, we will assume the trait space $\mathcal{X}$ to be unidimensional, i.e. an interval, to simplify the analysis and denote the trait as $x$ (but see Appendix C and Discussion).

Global invasion analysis. Let us extend the ideas presented in the previous 365 section to a continuum of competitors using geometrical envelopes of ZNGIs. This corresponds to species sorting, as all the strategies from the trait space are considered as potential invaders. Our aim here is to unify the different approaches and terminologies present in the literature (Meszéna and Metz, 1999, Chase and Leibold, 2003, Danger et al., 2008), provide some analytical and 370 geometrical properties of envelopes, and combine them with impact rays to construct community/eco-evolutionary bifurcation diagrams.

The concept of the envelope is easy to understand graphically and allows an extension of the invasion analysis to a continuum of competitors. Let us superimpose a large number of ZNGIs sampled from the trait space $\mathcal{X}$ and identify their discrete outer envelope in the sense of the previous section. When the number of ZNGI sampled tends toward infinity, this discrete envelope tends toward a (generally) smooth curve called the outer geometrical envelope of the ZNGI family (Fig. 3A). Note that to every point of this envelope, there is a tangent ZNGI. Mathematically, this condition of tangency formally defines the geometrical envelope. Indeed, the points $\boldsymbol{R}$ belonging to the envelope of a set of $\mathrm{ZNGI}_{x}$ from $\mathcal{X}$ locally satisfy:

$$
\begin{array}{r}
w(\boldsymbol{R}, x)=0 \\
\partial_{x} w(\boldsymbol{R}, x)=0
\end{array}
$$

where equation (5a) accounts for the fact that $\boldsymbol{R}$ has to belong to one of the 
A

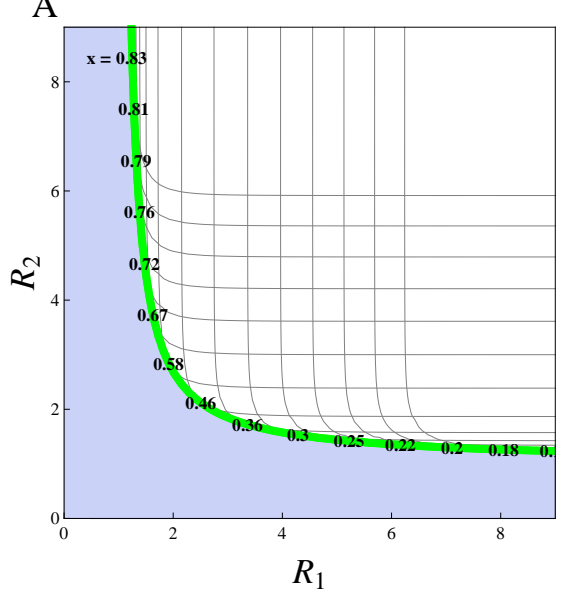

B

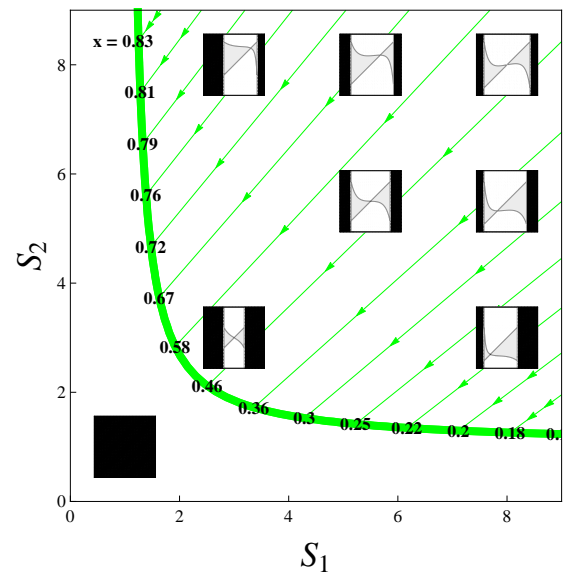

Figure 3: Graphical representation of a continuous set of populations consuming two resources $R_{1}$ and $R_{2}$ following the model of Box 1 in the interactive essential case $(\alpha=-5)$. A: the local envelope (thick, green) of the continuum of strategies $0 \leq x \leq 1$ has been displayed. A discrete subsample of strategies had their ZNGIs represented (thin, gray) and the corresponding trait value displayed at the point of contact with the envelope (thick, black). This local envelope appears to be global, as all the ZNGIs are situated above it. B: supply point mapping through the impact rays (arrowed lined). Ecologically, there is a single strategy outcompeting all the other ones when there is enough resources (supply points above the envelope). The optimal strategy tends to be specialized on $R_{1}$ as it becomes scarcer compared to $R_{2}$, and vice-versa. Contrary to the discrete case (Fig. 2A), there is no priority effect zones between neighbor strategies. PIPs (black $=$ resident can't exist, gray $=+$, white $=-$ ) have been displayed for comparison with adaptive dynamics framework (see section 2.3 and Fig. 5).

ZNGIs and equation (5b) imposes the supplementary condition of tangency.

Note that $\boldsymbol{R}$ is a priori considered independently of $x$ in the invasion analysis and thus not targeted by the partial derivative. In practice, an explicit equation of the envelope linking $R_{2}$ to $R_{1}$ can be obtained by eliminating $x$ from equations (5) or implicitly through a parametric equation.

Before moving on, let us emphasize the fact that eq. (5b) is a first order, that is, local criterion. As such, this does not insure that the envelope obtained with eq. (5b) has the global outer envelope behavior we are looking for. This situation can be understood by analogy with the problem of finding the global maximum of a differentiable function on a closed set. Setting its derivative equal to zero only locates the function's local extrema, which can be either maxima 


\section{A}

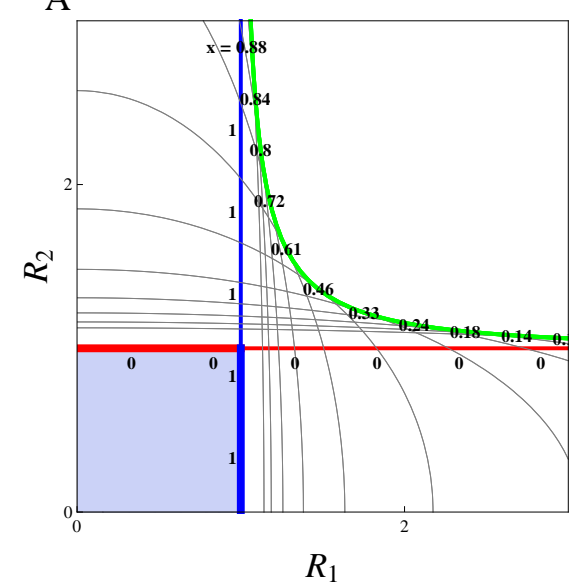

B

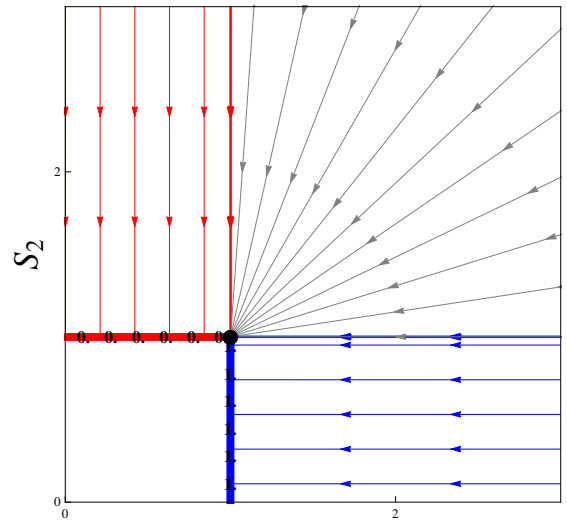

$S_{1}$

Figure 4: A: Local envelope (thick, green) for a continuum of strategies $0 \leq x \leq 1$ in the antagonistic case $(\alpha=2)$. A discrete subsample of strategies has been picked with their ZNGIs represented (thin, gray) and the corresponding trait value displayed at the point of tangency with the envelope (thick, black). The local envelope is an inner one and thus not part of the global one (thick), instead made of two portions of the boundary ZNGIs corresponding to the $R_{1}$ and $R_{2}$ specialists (resp. blue and red). B: supply point mapping via the impact rays (arrowed lined). There is room for coexistence in the 'cone' (gray) originating from the kink (black dot). Those results are very similar to the ones obtained in the discrete case (Fig. $2 \mathrm{~B})$.

or minima. The same happens with the local envelope obtained through eq. (5b). It can coincide with the global outer envelope we are looking for (Fig. 3A) and thus have its points $\boldsymbol{R}$ situated outside the whole ZNGI set as they satisfy $w(\boldsymbol{R}, x) \leq 0$ for any $x$. However, some portions of this envelope could also be local but not global while others could be inner envelopes, i.e. situated inside the whole ZNGI set with $w(\boldsymbol{R}, x) \geq 0$ for any $x$ (green segments in Fig. 4A). Those inner envelope portions have to be discarded in the global analysis as they are highly unstable, with every strategy able to invade. There is one last situation to consider. Back to our analogy of maximizing a function, the global maximum could also be situated on the boundaries of its domain and thus not be detected by setting its derivative equal to zero. In our case, it means that the global outer envelope could also be made of ZNGIs whose traits are located on the boundaries of $\mathcal{X}$ (red and blue segments in Fig. 4A). To conclude, the 
global invasion analysis is performed in the continuous case by putting together 410 the local envelope defined by eq. (5) and the boundary ZNGIs, and keeping their global outer envelope only (see Fig. 3, 4).

When solving eq (5), a singular trait $x^{*}(\boldsymbol{R})$ is associated with every point $\boldsymbol{R}$ of the envelope (see Box 1 and Fig. 3,4). This trait corresponds to the $\mathrm{ZNGI}_{x}$

415 that contributes to the envelope at that point $\boldsymbol{R}$. In the case of a global outer envelope, it means that this strategy $x$ is optimal for those specific regulating factor values $\boldsymbol{R}$, by outcompeting all the other strategies. Note that the global outer envelope can contain kinks where there is a discontinuity of $x^{*}(\boldsymbol{R})$ as $\boldsymbol{R}$ moves along the envelope. This means that two distinct ZNGIs are tangent to 420 the envelope at that specific point. As an important consequence, those are the only values of the regulating factors where globally stable coexistence is possible. Although kinks are generally plentiful in the discrete case between neighboring strategies (Fig. 1), the majority of those kinks usually vanish when the continuous limit is taken (Fig. 3). When globally stable coexistence of two different strategies from a continuum does occur, the associated kinks in the global envelope emerge at the self-intersections of the local envelope to which has been added its boundary ZNGIs when needed (Fig. 4). This is one of the major difference with the previous discrete approaches (Leibold, 1996, Chase and Leibold, 2003) which we will discuss later. These kinks make globally stable coexistence particularly easy to find and characterize graphically.

Supply point mapping. There is virtually no difference with the discrete strategy case. We only have to remember that there is a unique non-invadable strategy $x^{*}(\boldsymbol{R})$ associated with every point of the global envelope that is not a kink. 435 Plugging this relationship between the traits and the regulating factors into 
the renormalized impact vector $\tilde{\boldsymbol{I}}(\boldsymbol{R}, x)=\boldsymbol{I}(\boldsymbol{R}, x) / l_{i}$ allows us to draw the impact rays originating on the envelope points, thus performing the mapping from the ZNGI envelope to the supply point. The envelope thus behaves like a community-wide ZNGI, with its associated impact rays. The functioning of 440 the whole community can indeed be understood as a single entity that behaves like a single population. At a kink, the coexistence cone is obtained by plotting the impact rays associated with the two coexisting strategies, and the stability criterion (3) is checked as before.

${ }_{445}$ To summarize, the outcome of species sorting among a continuum of strategies can be seen from the community bifurcation diagram as a function of the supply points $\left(S_{1}, S_{2}\right)$. It is obtained by combining the envelope and impact rays through the following steps: (1) From the local envelope of the ZNGI continuum and its boundary ZNGIs, keep the global outer envelope. (2) For every 450 point of this envelope, identify the corresponding 'optimal' strategy. (3) Locate the regulating factor points corresponding to the 'empty' solutions (outside the envelope) and the potential coexistence solutions (the kinks). (4) Represent the supply point map by drawing impact rays from the envelope. (5) Identify the potentially globally stable coexistence 'cones' from the kinks.

455 2.3. Eco-evolutionary extension: link with adaptive dynamics

The global invasion analysis presented above considered that all the possible strategies from the trait space can invade the system and compete together. This explains why we have focused on determining the global envelope and discarded local but not global envelope portions. By doing so, we adopted an 460 'everything is everywhere' approach (Baas Becking, 1934, De Wit and Bouvier, 2006). At the opposite, the strategy space could be explored by evolving a single population through small mutation steps. In the previous section, we have 
presented a natural way to extend the graphical invasion analysis to a continuum of strategies by introducing the ZNGI envelope concept. As was shown

465 by Meszéna and Metz (1999), this framework naturally allows us to address eco-evolutionary equilibria of adaptive dynamics (Hofbauer and Sigmund, 1990, Geritz et al., 1997, 1998). In fact, the idea of addressing evolution with a graphical mutant invasion analysis can be traced back as far as the early developments of resource competition theory (MacArthur and Levins, 1964, MacArthur and

470 Wilson, 1967). Some methods based on graphical arguments in the trait space have already been developed to analyse evolutionary outcomes (Levins, 1962) and recently extended to fit in the density- and frequency-dependent context of adaptive dynamics (Rueffler et al., 2004, de Mazancourt and Dieckmann, 2004).

475 Here, we propose to further explore and describe the relationship between the ZNGI envelope and its geometrical properties and the evolutionary singular points and their classification. We will first provide some analytical results to support the intuition of Meszéna and Metz (1999). We will show how those results can be combined with the supply point mapping to provide a complete ${ }^{480}$ graphical characterization of the singular points. This approach makes it possible to draw eco-evolutionary bifurcation diagrams along the supply gradients. The whole approach relies on the observation that the growth function $w(\boldsymbol{R}, x)$, represented by $\mathrm{ZNGI}_{x}$, is actually the invasion fitness of a mutant $x$ in a residentdominated environment $\boldsymbol{R}(y)$. This means that the local envelope equations (5) 485 coupled with the supply point map given by the impact rays directly give the singular points of adaptive dynamics where the fitness gradient is zero. In the particular case of a one-dimensional trait $x$, we will show how the singular point classification is directly linked with the geometrical properties of the envelopes (but see Appendix C and discussion for the multidimensional case). 
Local invasion analysis. The local evolutionary stability (in the sense of noninvasibility) of a singular point can be characterized using the second derivative of the invasion fitness (Geritz et al., 1998):

$$
H(x)=\left[\frac{\partial^{2} w(\boldsymbol{R}(y), x)}{\partial x^{2}}\right]_{y=x} \equiv \frac{\partial^{2} w}{\partial x^{2}} .
$$

where $\partial^{2} R_{2} /\left.\partial R_{1}^{2}\right|_{E}$ and $\partial^{2} R_{2} /\left.\partial R_{1}^{2}\right|_{Z}$ are the second derivatives of respectively the envelope and the tangent ZNGI, and thus quantify their curvature. The difference between the two latter terms describes the relative curvature and thus position between the envelope and the tangent ZNGI: when negative, the envelope is located under the ZNGI set. Conversely, when this difference is positive, the envelope is located above the ZNGI set. The sign of $\partial w / \partial R_{2}$ translates this relative position along the $y$ axis in terms of relative fitness: when positive (as it is the case within our example, since $R_{2}$ is a resource), 'under' means 'outer envelope' $(w(\boldsymbol{R}, x) \leq 0$ for any nearby $x)$ and 'above' means 'inner envelope' $(w(\boldsymbol{R}, x) \geq 0$ for any nearby $x)$; conversely, when $\partial w / \partial R_{2}<0$, 'under' means 'inner' and 'above' means 'outer'. Eq. (7) makes the formal link with adaptive dynamics: outer envelope portions always correspond to ESS while the inner ones, which were discarded during the global invasion analysis, are associated with non-ESS. Inner envelope portions play an important role in the 
eco-evolutionary case as they can be associated with branching points (see below). Eq. (7) proves and generalizes the results of Meszéna and Metz (1999) to the case of non-linear ZNGIs, as the curvature of the ZNGI comes into play. As demonstrated for ecological coexistence, evolutionarily stable coexistence can be found at the self-intersections of the local envelope. We showed that its evolutionary stability is directly linked to the ones of its two constituting strategies: coexistence is evolutionarily stable if and only if situated at the intersection of two outer ZNGI envelope portions (see Appendix C for demonstration). In any case, ESS characterization of singular points only depends on ZNGIs.

A

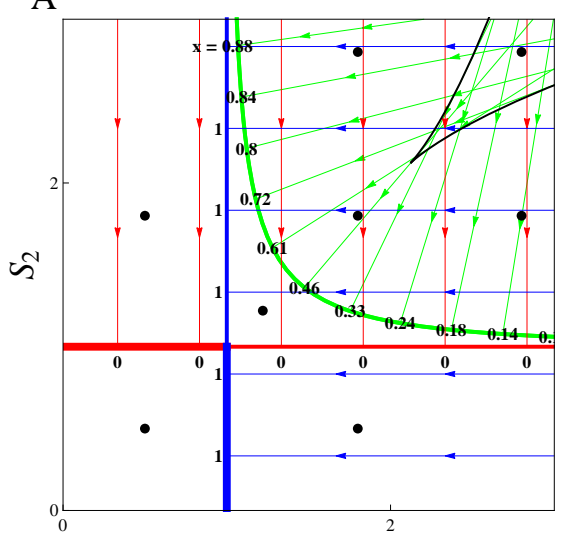

$S_{1}$

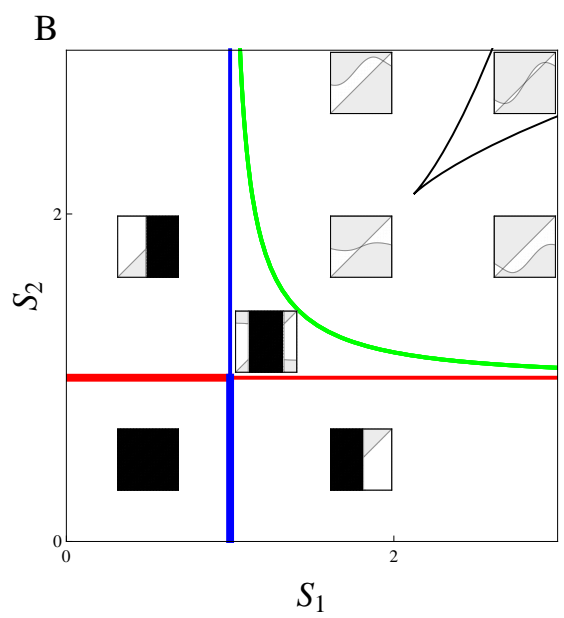

Figure 5: Eco-evolutionary bifurcation diagram along the supply gradients for the antagonistic case $(\alpha=2)$. A: full envelope composed of a non-ESS local portion (green) and two boundary ZNGIs (blue, red). The impact ray map (arrows, thin) helps visualizing the number and the properties of the singular points associated with a given supply point of the diagram. For example, every supply point inside the zone delimited by the impact ray envelope (black, thin) gives two boundary CSS, two repellers and one branching point. B: we only kept the global envelope and the skeleton of the impact ray map. Each zone delimited that way shares common properties that can be illustrated with PIPs (gray $=+$, white $=-$ ) being displayed at the exact location of their corresponding supply point (black dot, A). Invasion fitness is not defined for resident traits that do not allow the resident to exist (black). 
Supply point mapping. It is in fact possible to further characterize the singular

525

$$
\begin{aligned}
J(x) & =\frac{d}{d x}\left[\frac{\partial w(\boldsymbol{R}(y), x)}{\partial x}\right]_{y=x} \\
& \equiv\left(\frac{\partial}{\partial x}+\frac{\partial \boldsymbol{R}}{\partial x} \cdot \frac{\partial}{\partial \boldsymbol{R}}\right) \frac{\partial w}{\partial x}=H(x)+\frac{\partial \boldsymbol{R}}{\partial x} \cdot \frac{\partial}{\partial \boldsymbol{R}} \frac{\partial w}{\partial x}
\end{aligned}
$$

According to the adaptive dynamics classification of singular points, this Jacobian gives information about the singular point's convergence stability, telling if it is an attractor or a repeller for the 1D adaptive dynamics (Eshel, 1983, Geritz et al., 1998). More precisely, the singular point is said to be convergence stable if $J$ is negative. Note that this differs from the previous criterion based on the second derivative $H$ : for example, a singular point can be convergent stable but not evolutionarily stable, which is known as a branching point and can lead a single strategy to diversify into evolutionarily stable coexistence of two different strategies (Eshel, 1983, Metz et al., 1996, Geritz et al., 1997, 1998).

${ }_{535}$ It is possible to show the following relationship between $J$ and $H$ (see Appendix $\mathrm{C}$ for demonstration in the multidimensional trait case):

$$
J=\left(\left.\frac{\partial R_{1}}{\partial S_{1}}\right|_{\mathrm{Z}} /\left.\frac{\partial R_{1}}{\partial S_{1}}\right|_{\mathrm{E}}\right) H
$$

where $\partial R_{1} /\left.\partial S_{1}\right|_{Z}$ and $\partial R_{1} /\left.\partial S_{1}\right|_{E}$ describe how $R_{1}$ responds to its variation in supply, when $R_{1}$ moves along respectively the tangent ZNGI (ecological case, fixed strategy) and the envelope (eco-evolutionary case, adaptive strategy). The same relationship can be obtained for $R_{2}$ simply by replacing 1 by 2 from Eq. (9). First, note that $\partial R_{1} /\left.\partial S_{1}\right|_{Z}$ is non-negative for the usual consumer-resource or predator-prey interactions (but see Appendix B and C). This implies that $J$ and $H$ share the same signs when $\partial R_{1} /\left.\partial S_{1}\right|_{E}>0$ : an ESS is a CSS and a non${ }_{545}$ ESS is a repeller. Conversely, $J$ and $H$ have opposite signs when $\partial R_{1} /\left.\partial S_{1}\right|_{E}<0$ : an ESS is then non-convergent (Garden of Eden strategy) and a non-ESS is a 
branching point (Geritz et al., 1998). This last situation can be understood as follows: the eco-evolutionary feedback is so strong that the sign of the limiting factor response to supply variation, materialized by $\partial R_{1} /\left.\partial S_{1}\right|_{E}$, is completely 550 reversed compared to the purely ecological situation.

How can the sign of $\partial R_{1} /\left.\partial S_{1}\right|_{E}$ be read graphically? To see this, we need to introduce the notion of envelope of impact rays, following the same definition of envelope introduced earlier in the case of ZNGIs. Indeed, the set of impact 555 rays generated by moving the regulating factor point along the ZNGI envelope usually itself possesses an envelope (black curve, Fig. 5 and movie S1 in the Supplementary Material). A given impact ray will touch and be tangent to this envelope at a unique contact point. The line portion of the impact ray situated between its origin and this point corresponds to supply points satisfying $\partial R_{1} /\left.\partial s_{1}\right|_{E}>0$ while the other part corresponds to $\partial R_{1} /\left.\partial s_{1}\right|_{E}<0$. In general, crossing this envelope in the supply point space corresponds to the appearance or the disappearance of a pair of impact rays, i.e. singular points (see Fig. 5-6). This whole scheme can be understood as a way to use the supply point mapping to explicitly construct how the eco-evolutionary system responds to a local trait perturbation, following the ideas presented by Meszéna and Metz (1999).

We can understand how this works in practice by looking at our example (see also movies S2 and S3 in the Supplementary Material). In the interactive essential resource case, there is no impact ray envelope (see Fig. 3). According to the previous section, this means that $\partial R_{1} /\left.\partial s_{1}\right|_{E}>0$ and thus all ESS are CSS. The absence of an impact ray envelope also implies that impact rays never cross each other, which explains why there is never more than one singular point per supply point. In the antagonistic resource case, there is always an impact ray 
A

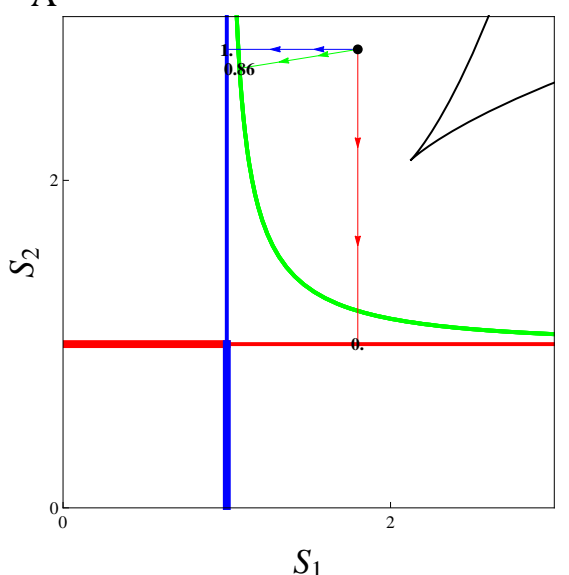

C

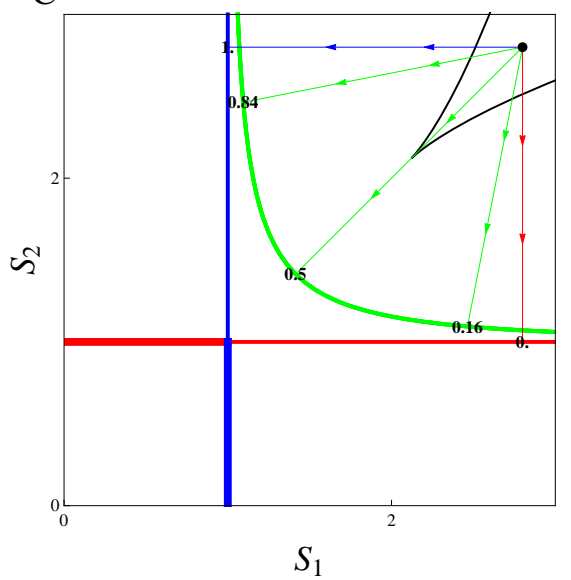

B

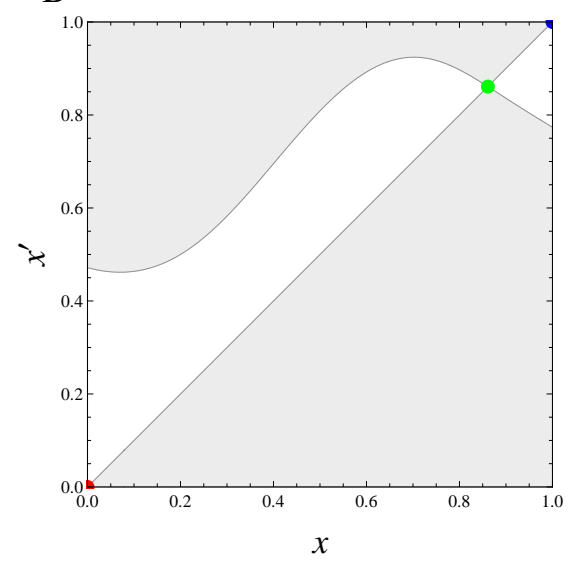

$\mathrm{D}$

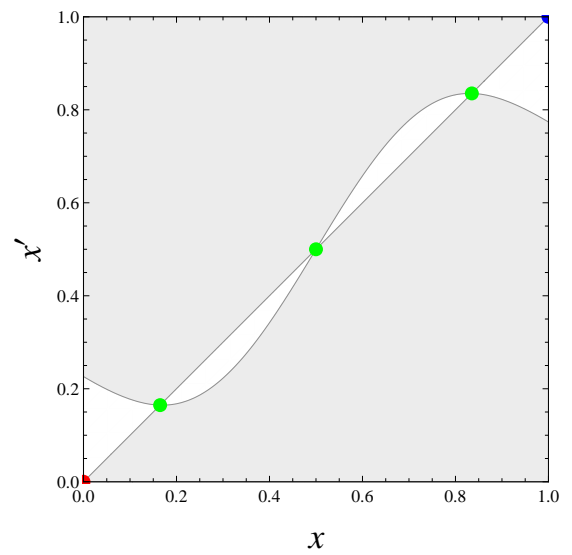

Figure 6: Details of the supply point map and its associated Pairwise Invasibility Plot (PIP) in the antagonistic case $(\alpha=2)$ for high but imbalanced (A,B) and balanced (C,D) resource supply, i.e. respectively $\boldsymbol{S}=(1.8,2.8)$ and $\boldsymbol{S}=(2.8,2.8)$. A: the impact rays map this supply point to three different eco-evolutionary equilibria on the envelope. The associated singular trait values are also displayed. B: PIP depicting the sign (gray $=+$, white $=-$ ) of the invasion fitness of a mutant with trait $x^{\prime}$ in a resident population with trait $x$. For a given resident strategy $x$, the success of the different invaders can be read along the corresponding vertical line. The limiting curves between the white and the gray regions correspond to invasion fitness equal to zero. Among them, the one-to-one line reminds us that the resident is at equilibrium. The eco-evolutionary fixed points are located at the intersection of this one-to-one line with the other contour or the boundary (colored dots). There are three of them here: one repelling singular point (green) and two boundary CSS $x=0$ (red) and $x=1$ (blue). The two latter strategies are locally non-invadable but not globally, as strategies different enough from them can invade. C,D: Contrary to the imbalanced case, this supply points gets mapped to three different points on the non-ESS envelope portion (green). Note that the middle impact ray corresponding to $x=0.5$ goes through its tangency point on the impact ray envelope (black, thin) before hitting the ZNGI envelope (C). As explained in the main text, this is the signature of this singular point being a branching point, as can be visualized on the PIP (D). 
envelope (see Fig. 5-6). Underneath the impact ray envelope, the same reasoning goes as for the interacting resource case. There is thus never more than one non-ESS repeller in that region (Fig. 6A,B). In contrast, above the impact ray envelope the impact ray map folds over on itself, leading to three singular points per supply point (Fig. 6C,D). Among them, the impact ray in the middle goes through its envelope tangency point before hitting the ZNGI envelope, which means that $\partial R_{1} /\left.\partial s_{1}\right|_{E}<0$ and it thus corresponds to a branching point (Fig. $6 \mathrm{D})$. The two other singular points are non-ESS repellers. In our example, the impact ray envelope thus delimits the region where non-boundary impact rays intersect. The emergence of alternative stable states as we cross the impact ray envelope is a well-known phenomenon in bifurcation theory, where it is referred to as a 'cusp catastrophe' (Strogatz, 2015).

As in the previous sections, evolutionary stable coexistence is only possible for supply points located between the two impact rays originating from a kink of the ZNGI envelope. Mutual invasibility, obtained by satisfying equation (3), 590 is also needed to ensure that this coexistence is ecologically stable. Moreover, when polymorphism is saturated (as many distinct strategies as regulating factors) we have $J=H$ for each of the two coexisting strategies (see Appendix C). Thus, evolutionarily stable coexistence is automatically convergence stable coexistence and further evolutionary branching is impossible, in accordance with the CEP (Meszéna and Metz, 1999). This last result is consistent with a recent study of saturated polymorphism (Kisdi and Geritz, 2016).

To summarize, an eco-evolutionary bifurcation diagram along the regulating factors supply can be obtained in the unidimensional trait case through the following steps: (1) Draw the ZNGI envelope. (2) Identify the ESS and non- 


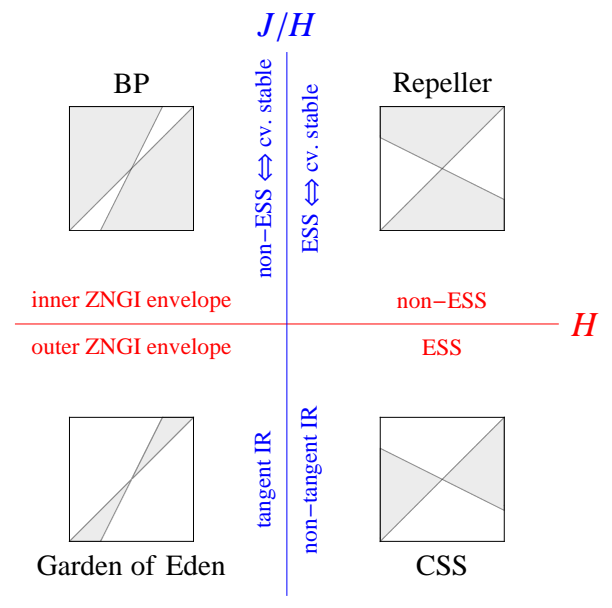

Figure 7: Correspondance between the classification of singular strategies and their graphical characterization in the standard consumer-resource or predator-prey case where $I_{2} . \partial w / \partial R_{2}>$ 0, e.g. Schreiber and Tobiason's (2003) or Leibold's (1996). The classification has been adapted and simplified from Geritz et al. (1997). 'tangent IR' and 'non-tangent IR' stand for 'impact ray tangent to its envelope' and 'impact ray not tangent to its envelope', while 'cv.' stands for 'convergence'. The case where $I_{2} . \partial w / \partial R_{2}<0$ (e.g. nitrogen fixing) is obtained by permuting 'tangent IR' and 'non-tangent IR' from the figure.

ESS portions (given by the envelope's relative position with ZNGIs) and add boundary ZNGIs if necessary. (3) Draw the impact ray envelope and a subset of impact rays to represent the supply point map. (4) If there is an evolutionarily stable self-intersection of the ZNGI envelope, draw the coexistence cone. (5) Identify the different regions delimited and the properties of the associated singular points (Fig. 7). Note that superimposing a Pairwise Invasibility Plot (PIP) (Geritz et al., 1997) for every region of the diagram helps in visualizing the eco-evolutionary characteristics of the system, like the number and properties of the singular points and mutual invasibility associated with singular dimorphism (Fig. 3-6). Indeed, those conserved singular point characteristics make PIPs qualitatively similar inside a given region of the diagram.

\section{Box 1: Schreiber and Tobiason consumer-resource model}

Schreiber and Tobiason (2003) studied the evolutionary ecology of $n$ consumer populations feeding on two resources with densities $R_{1}$ and $R_{2}$. Their model is a particular case of model (1) from the main text, 
with:

$$
\begin{aligned}
& w_{i}\left(R_{1}, R_{2}\right)=g_{i}\left(R_{1}, R_{2}\right)-m \\
& g_{i}\left(R_{1}, R_{2}\right)=\left[\left(x_{i} R_{1}\right)^{\alpha}+\left(\left(1-x_{i}\right) R_{2}\right)^{\alpha}\right]^{\frac{1}{\alpha}}
\end{aligned}
$$

where $x_{i}$ and $1-x_{i}$ account for investment in acquisition of respectively resource 1 and 2 (note this implies a linear trade-off), $m$ is the constant per capita mortality rate and $\alpha$ controls the shape of the interaction between resources. Following Tilman's $(1980,1982)$ classification of resources relations, $\alpha<0$ represents interactive essential resources (both necessary and slightly better in balanced proportions), $0<\alpha<1$ represents complementary resources (substitutable but better in balanced proportions), $\alpha=1$ represents perfectly substitutable resources, and $\alpha>1$ represents antagonistic resources (substitutable but worse in balanced proportions). The limiting cases $\alpha \rightarrow-\infty$ and $\alpha \rightarrow+\infty$ lead to respectively essential and switching resources (growth is limited by respectively the most limiting and the most abundant resource). The growth rate (11) is thus an elegant mathematical way to control the nutritional interaction between the resources. The intrinsic resource dynamics follows chemostat dynamics as in eq. (1b) with $l_{1}=l_{2}$. Finally, population $N_{i}$ influences the resources dynamics in model (1) through its impact vector. For $\alpha<1$, we retain the mass action law used by Schreiber and Tobiason (2003):

$$
\left(I_{i 1}, I_{i 2}\right)=-\left[x_{i} R_{1},\left(1-x_{i}\right) R_{2}\right]
$$

This describes purely random encounters and removal of both resources, proportionally to their densities through acquisition rates. This con- 
sumption process does not satisfy conservation of mass in general, as $I_{i 1}+I_{i 2} \neq g_{i}$ for $\alpha \neq 1$. Thus, removal of a certain resource density does not translate into an equivalent consumer growth. In the case of antagonistic resources, this situation would describe nutritional antagonism during the assimilation process, like synergistic effects of toxic compounds (Tilman, 1980). For this reason, we rather used the following impacts in the $\alpha \geq 1$ case:

$$
\left(I_{i 1}, I_{i 2}\right)=-g_{i}^{1-\alpha}\left[\left(x_{i} R_{1}\right)^{\alpha},\left(\left(1-x_{i}\right) R_{2}\right)^{\alpha}\right]
$$

Conservation of mass is here satisfied, and antagonism comes from the foraging strategy of the consumer itself. It describes the behavioral switching of a predator, focusing disproportionately on its most abundant prey (Murdoch, 1969). This situation can emerge when resources are spatially distributed (Murdoch et al., 1975) or through the formation of a search image (Pietrewicz and Kamil, 1979, Dukas and Kamil, 2001).

As explained in the main text, the ZNGI of a given population $i$ is obtained by setting $g_{i}\left(R_{1}, R_{2}\right)-m=0$. Its concavity is controlled by the sign of $\alpha-1$, as can be visualized in Fig. 1: the antagonistic case $(\alpha>1)$ give concave ZNGIs while complementary and interactive essential $(\alpha<1)$ gives convex ones. Moreover, computing the equation of the ZNGIs envelope through eq. (5) gives the eco-evolutionary singular points of the system. Solving them in this particular case leads to the following implicit envelope equation:

$$
\left(R_{1}^{\frac{\alpha}{1-\alpha}}+R_{2}^{\frac{\alpha}{1-\alpha}}\right)^{\frac{1-\alpha}{\alpha}}-m=0
$$


This also gives the expression of the singular trait as a function of the resource level:

$$
x\left(R_{1}\right)=\left(\frac{R_{1}}{m}\right)^{\frac{\alpha}{1-\alpha}}
$$

When substituted into the impact vector expression, this gives the impact map linking supply points to the corresponding singular points. The resulting ZNGI envelopes and associated impact maps are represented in Fig. 3-5. In the antagonistic case, the envelope had to be supplemented with the boundary ZNGIs. Those are the horizontal and vertical lines going through the point $(1,1)$, and correspond to the two specialist strategies $x=0$ and $x=1$.

\section{Discussion}

In this paper, we show how the graphical approach of contemporary niche theory can be extended to a continuum of strategies to give insights into community assembly processes and eco-evolutionary dynamics along environmental gradients. In section 2.1, we reviewed the graphical approach by providing a general step-by-step recipe to create ecological bifurcation diagrams along environmental gradients. In section 2.2 , we adapted this recipe to the situation of a continuum of competitors using geometrical envelopes, enabling us to study community assembly from a large species pool. Finally, in section 2.3 we demonstrated that combining this extension of the graphical approach with the adaptive dynamics framework leads to eco-evolutionary bifurcation diagrams summarizing the various possible evolutionary outcomes of the system.

\subsection{Extension to structured populations}

In this paper, we have restricted our attention to unstructured populations, demographically and spatially. This was done for the sake of simplicity, as 
there is no further complication to apply this graphical method to the case of linearly structured populations, that are defined by their dynamics satisfying $d \boldsymbol{N}_{i} / d t=\boldsymbol{w}_{i}\left(R_{1}, R_{2}, \ldots, R_{p}\right) \boldsymbol{N}_{i}$, where the $\boldsymbol{N}_{i}$ are vectors of abundances at the different states (ages, sizes, or patches, for example) and $\boldsymbol{w}_{i}$ the net growth matrix (Leslie, 1948, Caswell, 2001). In this case, the ZNGI equation is obtained by setting the largest eigenvalue of the net growth matrix equal to zero (Loreau and Ebenhöh, 1994, Schellekens et al., 2010, Haegeman and Loreau, 2015). The corresponding eigenvector determines the population structure at equilibrium, thus reducing the impact on the regulating factors to a one-dimensional problem

${ }_{640}$ similar to the unstructured case. Conversely, there is no such general rule in the case of non-linear population structure, e.g. two-sex models (but see Szilágyi and Meszéna 2009, Barabás et al. 2014a). However, it may still be possible to define a ZNGI, as is the case for the Droop model after a quasi steady state approximation (Klausmeier et al., 2004). Therefore, it is generally possible to ${ }_{645}$ apply the envelope method to study the eco-evolution of structured populations.

\subsection{Extension to higher trait space dimensions}

In this paper, we restricted the presentation of the eco-evolutionary graphical method to the case of a unidimensional trait space. However, it is still possible to define a local envelope in the general case of a trait space with dimension $k$ ${ }_{650}$ by replacing the trait derivative of eq. (5) by a $k$-dimensional trait gradient. This $k$-dimensional trait envelope can be seen as the outcome of a recursive scheme consisting of taking successively $k$ times the envelope along every trait vector component, starting from the ZNGI multidimensional set. In any case, the ZNGI envelope keeps the property of being a unidimensional curve. This has important ecological consequences, as was pointed out by a more general result of Meszéna and Metz (1999): the singular strategies are necessarily contained in a $p-1$ sub-manifold of the trait space, where $p$ is the number of regulating 
factors. This means that there is only a specific set of trait combinations that can be evolutionarily stable strategies, all the other ones being automatically discarded whatever the supply point. This introduces correlations between the traits of organisms that could ever be observed. This process can be thought as a pure 'competitive filtering' as it only relies on the invasion analysis. It is thus completely independent of the details of the embedding environment and as such, a very general result. Note that Tilman's (1982) $R^{*}$ rule is a special case of this result, where a single limiting factor usually leads to a unique singular strategy, or at least a countable number, whatever the supply point. Can a singular point still be characterized locally from the geometry of the envelope in the multidimensional case? Even if the relationships (7) and (9) can be extended to the $k$-dimensional case (see Appendix C), they do not give enough information to perform this full characterization.

To conclude, a ZNGI envelope can be obtained for any dimension of the trait space. It selects from the full trait space the strategies that are singular and represents graphically their competitive ability. Their local characteristics can not be deduced from simple graphical properties, but global evolutionary stability remains easy to identify.

\subsection{From local to global invasibility}

The two perspectives presented in sections 2.2 and 2.3 can be seen as two opposite but complementary pictures. The first, sometimes called the 'everything is everywhere' picture, assumes that all the imaginable strategies from the trait space have a chance to invade the system. The details of the creation and maintenance of this diversity of invaders is simply assumed (Sauterey et al., 2015). This can be seen as the existence of a hyperdiverse regional species pool - the system being embedded in an heterogenous and connected landscape - or with 
invasion of a local neighborhood of strategies around the resident, as mutations are small. In this approach, evolution can be 'trapped' at a local-only ESS, and diversification from a monomorphic population only emerges from a branching point. The two approaches can lead to similar bifurcation diagrams, as it is the case for interactive essential resources in the example (Fig. 3) where all the local singular points are global CSS. However, the presence of locally non-ESS envelope portions lead to significant differences between the two pictures. In the case of antagonistic resources from our example, the local analysis identifies regions of priority effect between two locally stable specialists and a branching point (Fig. 5B, 6D). Those details do not matter in the global analysis, replaced by evolutionarily stable coexistence of the two specialists (Fig. 4B). The link between the two frameworks can be seen from the PIPs in the second picture, as shown in Fig. 5B: evolution under small mutations can be read on the diagonal neighborhood, but information about invasion of any strategy is also available away from it. Another remark clear in the previous example is that there is no reason for the evolutionarily stable coexistence region and the branching region to coincide. Evolutionarily and convergence stable dimorphism are indeed possible in the absence of branching, thus emerging through invasion or 'macro-mutation' (Geritz et al., 1999) (Fig. 5B). An example of this situation was discussed by Wolf et al. (2007), Massol and Crochet (2008), Wolf et al. (2008). Conversely, branching can happen in a region where evolutionary stable coexistence is not possible: one of the two morphs would inevitably experience evolutionary suicide along its eco-evolutionary trajectory (Matsuda and Abrams, 1994, Rankin and López-Sepulcre, 2005, Parvinen, 2005). Local and global approaches are the two extremes of a general invasion analysis picture that can be visualized by combining local and global bifurcation diagrams and 
associating them with PIPs.

\subsection{Up- and downscaling with ZNGI envelopes}

We have explained in section 2.2 how the envelope approach allows one to scale up from the population to the community level to sort out the best competitor from a continuum of strategies. It is also possible to scale down from the population to the individual level in the context of phenotypic plasticity (Tilman, 1982). Indeed, dynamical allocation in response to environmental cues could allow an individual to explore the trait space in search of the optimal strategy, in the sense of competition. The envelope approach gives a practical tool to do so: from all the accessible behaviors represented by the continuous set of ZNGIs, the optimization procedure only retains their envelope, which can be seen as the new integrated ZNGI of the plastic individuals. Adding the family of adaptive impact rays, a direct parallel can be drawn between populations of plastic and non-plastic individuals (Tilman, 1982, Schade et al., 2005). The only difference is that the traits of the former are optimized, thus depending on the limiting factor values. A corollary is that a variety of adaptive ZNGI shapes can arise from simple non-plastic ones. As such, the envelope method provides a practical procedure to navigate through levels of organization by taking into account adaptation and flexibility, from individuals to communities (Smith et al., 2011, Norberg, 2013).

\subsection{Coevolution from distinct functional groups and discontinuous mutations}

The envelope approach is easy to apply to the evolution of $n$ independent guilds, for example from different functional groups. Those guilds must share some regulating factors to be able to interact, but can belong to completely different trait spaces, or be bound by completely distinct trade-offs. Each guild would lead to its own eco-evolutionary envelope, that can then be compared as if they were ZNGIs in the standard discrete invasion analysis (section 
2.1), as emphasized in the previous subsection. Examples could include plants and decomposers along a material cycle (Loreau, 1998a), nitrogen-fixing and non-nitrogen-fixing phytoplankton (Boushaba and Pascual, 2005, Agawin et al., 2007) or pairs of cooperators (de Mazancourt and Schwartz, 2010). This picture could also allow one to study the global eco-evolutionary outcomes of a strategy composed of a combination of continuous and discrete traits. From the previous point of view, this means allowing a jump from one functional group to another through discontinous mutations. With a unidimensional bounded continuous trait, invasion analysis could still be accurately depicted coupling several PIPs together into a 'meta-PIP'.

\subsection{From discrete to continuous set of strategies}

The continuous approach presented in section 2.2 clarifies some results identified in the finite number of strategies context. Indeed, the study of species sorting along environmental gradients has usually been addressed using a large but finite number of competitors. This led some authors to conclude that "coexistence was more likely among the most similar form" (Leibold, 1996). Fig. 1A from the interactive essential case contains the signature of this phenomena, as neighboring ZNGIs cross at potential coexistence points. This does not lead to coexistence here because of our choice of impact vectors but the idea is the same. However, we argue that this pattern of coexistence of similar forms is degenerate and corresponds to some kind of nearly 'neutral coexistence', i.e. not ecologically robust sensu Meszéna et al. (2006). This can been seen when looking at the continuous limit in Fig. 3. The coexistence points identified earlier have vanished, as neighboring ZNGIs are now infinitely close. In eco-evolutionary terms, this coexistence is not evolutionarily robust as evolution tends to destroy it. Yet, this does not mean that evolutionarily stable coexistence can not hap${ }_{765}$ pen in the continuous limit, as we have seen in the antagonistic case (Fig. 4). It 
is, however, far less common as it relies on self-intersection of the local envelope, but more robust. Note that those differences depends heavily on the topology of the strategy space: disconnected in the finite case versus connected after the continuous limit. This is in practice related to the question of the existence of infinitely many intermediate forms between different strategies.

If this pattern of coexistence vanishes at the continuous limit, it still leaves a signature on the invasion dynamics. More precisely, whether the potential coexistence points lead to stable coexistence or priority effect influences the shape of the PIPs around the singular points. This can be seen in the interactive essential resource example. For high resource supplies, priority effect between neighboring strategies (Fig. 1A) translates into CSS strategies that cannot directly invade their neighborhood and are only attained monotonically through ever-decreasing evolutionary steps (see PIPs on Fig. 3B). This is one of the eight singular strategy types identified by the adaptive dynamics classification (Geritz et al., 1997). On the contrary, we predict that coexistence between neighboring strategies in the finite strategy case would lead to CSS strategies able to invade their neighborhood. Giving a mathematical proof of this is out the scope of this paper. However, those results are intuitive, as priority effects between neighboring strategies indicate that they are protected from invasion by the other strategies. To conclude, while neighboring coexistence or priority effect vanish when the continuous limit is taken, they leave their signature on the eco-evolutionary characteristics of the singular points.

\subsection{Importance of the regulating factor space}

For a given strategy, the corresponding ZNGI summarizes its competitive ability. This is measured in the regulating factor space, where the competitiveness of different strategies can be compared. In the case of a single regulating 
factor, the ZNGI reduces to one number, so all the strategies can be ordered and compared without ambiguity. This result is known as Tilman's $R^{*}$ rule (Hsu et al., 1977, Tilman, 1982, Grover, 1997, Chase and Leibold, 2003) and leads to a pessimization principle in the eco-evolutionary case (Metz et al., 2008). This strict ordering is in general impossible with more regulating factors: invasion analysis with the envelope is the closest equivalent to that rule. Optimization is now multi-objective, so a Pareto front is needed to find the optimal strategies, and this role is played by the envelope. This is why the regulating factor space is so central: it controls species sorting and adaptation through this multiobjective optimization. However, the map between a strategy from the trait space and its ZNGI in the regulating factor space is non-trivial. For example, constraints on organisms encoded by trade-offs are usually inferred at the trait level. Yet, nothing ensures that they efficiently translate into a trade-off in competitive ability of the ZNGI set in the regulating factor space. Another effect of this map is to control the presence of evolutionarily stable coexistence. Indeed, it relates the geometrical characteristics of the ZNGI envelope to the ones of the trait space. For example, some trait space geometries lead to kinked global envelopes and thus potentially to evolutionarily stable coexistence while other do not.

\subsection{Evolution of resource use}

Applying the envelope method to Schreiber and Tobiason's (2003) resource competition model allowed us to confirm and visualize their results. Moreover, we could specify how the number of singular points and their properties depended on the resource supplies through bifurcation diagrams. For $\alpha<1$, there is always a single generalist CSS, under the condition that there is a sufficient supply of resources (Fig. 3). The antagonistic case $(\alpha>1)$ was further characterized with the help of the impact ray map and its envelope (Fig. 5). 
First, there is always a zone of supply points for which evolutionary branching is possible. This is true for both the choice of impact vector expressions used by Schreiber and Tobiason (2003) and the modification we proposed. However, this zone is pushed infinitely far away from the envelope for $\alpha \rightarrow \infty$ when using eq. (12) while it stays close to it when using eq. (13). The former is consistent with Schreiber and Tobiason's (2003) observations. The latter states that branching is still possible on highly antagonistic resources if switching is more abrupt. Note that branching demands a sufficiently large and balanced supply of the two resources to take place. Moreover, this branching point, when it exists, is always unique and separated from the boundary attracting strategies by two repellers. Also note that it is possible not to have any singular point but still one or two attractive boundaries for low enough resources.

The consumer-resource model of the example also gives some insights in the case of strictly essential ressources. This corresponds to the limit obtained when ${ }_{835} \alpha \rightarrow-\infty$, leading to a growth rate (11) following Liebig's Law of the Minimum and an associated L-shaped ZNGI (León and Tumpson, 1975, Tilman, 1982). It is actually in this context that the use of ZNGI envelopes first appeared (Tilman, 1988) and later spread (Schade et al., 2005, Klausmeier et al., 2007, Danger et al., 2008). The standard approach consists in getting the envelope equation simply 840 by tracking the position of the ZNGI corner. The first order criterion (5b) is not only unnecessary in this case, it actually fails to give the envelope equation when applied directly, as the L-shaped ZNGI is non-differentiable at its corner. However, this problem can be worked around by taking the limit when $\alpha \rightarrow-\infty$ of the general envelope equation (14), leading to a consistent result. Contrary 845 to the standard approach, our method is easy to generalize to a trait space of arbitrary dimension. It could thus be used to further investigate the evolution 
of consumers feeding on essential resources (Klausmeier et al., 2004, Shoresh et al., 2008).

\subsection{Conclusion and perspectives}

In this paper, we presented a graphical approach based on geometrical envelopes that can be used to perform invasion analysis and supply point mapping with a continuum of interacting strategies. We showed how relevant this technology is to two biological pictures, namely species sorting and adaptive dynamics, paving the way for an 'evolutionary theory of the niche' (Holt, 2009). Because of its generality, this approach could be applied to investigate a variety of ecological situations: the evolution in a diamond-shaped food web (Leibold, 1996), cooperation through trading (de Mazancourt and Schwartz, 2010), informed dispersal (Haegeman and Loreau, 2015), nitrogen fixing (Agawin et al., 2007) or niche construction (Kylafis and Loreau, 2011), for example.

\section{Acknowledgments}

We thank Thomas Aubier, Géza Meszéna and an anonymous reviewer for valuable comments on the manuscript. This work was supported by a Grant from Simons Foundation (343149, C.A.K.). T.D., C.A.K. and F.M. were able to work together thanks to the "Stoichiometry in metaecosystems" Working Group

865 at the National Institute for Mathematical and Biological Synthesis, sponsored by the National Science Foundation, the U.S. Department of Homeland Security, and the U.S. Department of Agriculture through NSF Award \# EF-0832858, with additional support from The University of Tennessee, Knoxville.

\section{References}

Adler, P. B., Dalgleish, H. J., and Ellner, S. P. (2012). Forecasting plant community impacts of climate variability and change: when do competitive interactions matter? Journal of Ecology, 100(2):478-487. 
Agawin, N. S. R., Rabouille, S., Veldhuis, M. J. W., Servatius, L., Hol, S., van Overzee, H. M. J., and Huisman, J. (2007). Competition and facilitation between unicellular nitrogen-fixing cyanobacteria and non-nitrogen-fixing phytoplankton species. Limnology and Oceanography, 52(5):2233-2248.

Armstrong, R. A. (1979). Prey species replacement along a gradient of nutrient enrichment: a graphical approach. Ecology, 60(1):76-84.

Baas Becking, L. G. M. (1934). Geobiologie of Inleiding Tot de Milieukunde. Van Stockum, Den Haag.

Barabás, G., Meszéna, G., and Ostling, A. (2014a). Fixed point sensitivity analysis of interacting structured populations. Theoretical Population Biology, 92:97-106.

Barabás, G., Pásztor, L., Meszéna, G., and Ostling, A. (2014b). Sensitivity analysis of coexistence in ecological communities: theory and application. Ecology Letters, 17(12):1479-1494.

Boushaba, K. and Pascual, M. (2005). Dynamics of the 'echo' effect in a phytoplankton system with nitrogen fixation. Bulletin of Mathematical Biology, 67(3):487-507.

Caswell, H. (2001). Matrix Population Models: Construction, Analysis, and Interpretation. Sinauer Associates, Sunderland, MA.

Chase, J. M. and Leibold, M. A. (2003). Ecological Niches: Linking Classical and Contemporary Approaches. University of Chicago Press, Chicago.

Danger, M., Daufresne, T., Lucas, F., Pissard, S., and Lacroix, G. (2008). Does 895 Liebig's law of the minimum scale up from species to communities? Oikos, 117(11):1741-1751. 
Daufresne, T. and Hedin, L. O. (2005). Plant coexistence depends on ecosystem nutrient cycles: extension of the resource-ratio theory. Proceedings of the National Academy of Sciences, 102(26):9212-9217.

de Mazancourt, C. and Dieckmann, U. (2004). Trade-off geometries and frequency-dependent selection. The American Naturalist, 164(6):765-778.

de Mazancourt, C. and Schwartz, M. W. (2010). A resource ratio theory of cooperation. Ecology Letters, 13(3):349-59.

De Wit, R. and Bouvier, T. (2006). 'Everything is everywhere, but, the environment selects'; what did Baas Becking and Beijerinck really say? Environmental Microbiology, 8(4):755-758.

Dieckmann, U. and Doebeli, M. (1999). On the origin of species by sympatric speciation. Nature, 400(6742):354-357.

Dieckmann, U. and Ferrière, R. (2004). Adaptive dynamics and evolving biodiversity. In Evolutionary Conservation Biology, pages 188-224. Cambridge University Press, Cambridge, UK.

Dieckmann, U. and Law, R. (1996). The dynamical theory of coevolution: a derivation from stochastic ecological processes. Journal of Mathematical Biology, 34(5-6):579-612.

Dieckmann, U. and Metz, J. A. J. (2006). Surprising evolutionary predictions from enhanced ecological realism. Theoretical Population Biology, 69(3):263281.

Dobzhansky, T. (1973). Nothing in biology makes sense except in the light of evolution. The American Biology Teacher, 35(3):125-129.

Dukas, R. and Kamil, A. C. (2001). Limited attention: the constraint underlying search image. Behavioral Ecology, 12(2):192-199. 
Eppley, R. W. (1972). Temperature and phytoplankton growth in the sea. Fishery Bulletin, 70(4):1063-1085.

Eshel, I. (1983). Evolutionary and continuous stability. Journal of Theoretical Biology, 103:99-111.

Gause, G. F. (1934). The struggle for existence. Williams \& Wilkins Company, Baltimore, MD.

Geritz, S., Metz, J. A. J., Kisdi, É., and Meszéna, G. (1997). Dynamics of adaptation and evolutionary branching. Physical Review Letters, 78(10):20242027.

Geritz, S. A., van der Meijden, E., and Metz, J. A. (1999). Evolutionary dynamics of seed size and seedling competitive ability. Theoretical Population Biology, 55(3):324-343.

Geritz, S. A. H., Kisdi, É., Meszéna, G., and Metz, J. A. J. (1998). Evolutionarily singular strategies and the adaptive growth and branching of the evolutionary tree. Evolutionary Ecology, 12(1):35-57.

Gerla, D. J., Vos, M., Kooi, B. W., and Mooij, W. M. (2009). Effects of resources and predation on the predictability of community composition. Oikos, 118(7):1044-1052.

${ }_{940}$ Grant, P. R. and Grant, B. R. (2006). Evolution of character displacement in Darwin's finches. Science (New York, N.Y.), 313(5784):224-226.

Grover, J. P. (1995). Competition, herbivory and enrichment: nutrient-based models for edible and inedible plants. The American Naturalist, 145(5):746774.

${ }_{945}$ Grover, J. P. (1997). Resource Competition. Chapman \& Hall, London. 
Guill, C. (2009). Alternative dynamical states in stage-structured consumer populations. Theoretical population biology, 76(3):168-78.

Gyllenberg, M. and Meszéna, G. (2005). On the impossibility of coexistence of infinitely many strategies. Journal of Mathematical Biology, 50:133-160.

Haegeman, B. and Loreau, M. (2015). A graphical-mechanistic approach to spatial resource competition. The American Naturalist, 185(1):1-13.

Hairston, N. G., Ellner, S. P., Geber, M. A., Yoshida, T., and Fox, J. A. (2005). Rapid evolution and the convergence of ecological and evolutionary time. Ecology Letters, 8(10):1114-1127.

Hofbauer, J. and Sigmund, K. (1990). Adaptive dynamics and evolutionary stability. Applied Mathematics Letters, 3(4):75-79.

Holt, R. D. (1977). Predation, apparent competition, and the structure of prey communities. Theoretical Population Biology, 12(2):197-229.

Holt, R. D. (2009). Bringing the Hutchinsonian niche into the 21st century: ecological and evolutionary perspectives. Proceedings of the National Academy of Sciences, 106:19659-19665.

Holt, R. D., Grover, J. P., and Tilman, D. (1994). Simple rules for interspecific dominance in systems with exploitative and apparent competition. The American Naturalist, 144(5):741-771.

Hsu, S. B., Hubbell, S., and Waltman, P. (1977). A mathematical theory for single-nutrient competition in continuous cultures of micro-organisms. SIAM Journal on Applied Mathematics, 32(2):366-383.

Huisman, J. and Weissing, F. J. (1999). Biodiversity of plankton by species oscillations and chaos. Nature, 402(6760):407-410. 
Kéfi, S., Eppinga, M. B., de Ruiter, P. C., and Rietkerk, M. (2010). Bistability and regular spatial patterns in arid ecosystems. Theoretical Ecology, 3(4):257269.

Kisdi, É. and Geritz, S. A. H. (2016). Adaptive dynamics of saturated polymorphisms. Journal of Mathematical Biology, 72(4):1039-1079.

975 Klausmeier, C. A., Litchman, E., Daufresne, T., and Levin, S. A. (2004). Optimal nitrogen-to-phosphorus stoichiometry of phytoplankton. Nature, 429(6988):171-174.

Klausmeier, C. A., Litchman, E., and Levin, S. A. (2007). A model of flexible uptake of two essential resources. Journal of Theoretical Biology, 246(2):27889.

Kneitel, J. M. and Chase, J. M. (2004). Trade-offs in community ecology: linking spatial scales and species coexistence. Ecology Letters, 7(1):69-80.

Kylafis, G. and Loreau, M. (2011). Niche construction in the light of niche theory. Ecology Letters, 14(2):82-90.

${ }_{985}$ Lavorel, S. and Garnier, E. (2002). Predicting changes in community composition and ecosystem functioning from plant traits: revisting the Holy Grail. Functional Ecology, 16(5):545-556.

Lavorel, S. and Grigulis, K. (2012). How fundamental plant functional trait relationships scale-up to trade-offs and synergies in ecosystem services. Journal of Ecology, 100(1):128-140.

Leibold, M. A. (1996). A graphical model of keystone predators in food webs: trophic regulation of abundance, incidence, and diversity patterns in communities. The American Naturalist, 147(5):784-812. 
Leibold, M. A., Holyoak, M., Mouquet, N., Amarasekare, P., Chase, J. M.,

995 M., and Gonzalez, A. (2004). The metacommunity concept: a framework for multi-scale community ecology. Ecology Letters, 7(7):601-613.

Leimar, O. (2009). Multidimensional convergence stability. Evolutionary Ecology Research, 11(2):191-208.

1000

Lester, S. E., Costello, C., Halpern, B. S., Gaines, S. D., White, C., and Barth, J. A. (2013). Evaluating tradeoffs among ecosystem services to inform marine spatial planning. Marine Policy, 38:80-89.

Levin, S. A. (1970). Community equilibria and stability, and an extension of the competitive exclusion principle. The American Naturalist, 104(939):413-423.

1010

Levins, R. (1962). Theory of fitness in a heterogeneous environment. I. The fitness set and adaptive function. The American Naturalist, 96(891):361-373.

Litchman, E. and Klausmeier, C. A. (2008). Trait-based community ecology of phytoplankton. Annual Review of Ecology, Evolution, and Systematics, 39(1):615-639.

1015

Litchman, E., Klausmeier, C. A., Schofield, O. M., and Falkowski, P. G. (2007). The role of functional traits and trade-offs in structuring phytoplankton communities: scaling from cellular to ecosystem level. Ecology Letters, 10(12):1170-1181. 
Loreau, M. (1998a). Biodiversity and ecosystem functioning: a mechanistic model. Proceedings of the National Academy of Sciences of the United States of America, 95(10):5632-5636.

Loreau, M. (1998b). Ecosystem development explained by competition within and between material cycles. Proceedings of the Royal Society B: Biological Sciences, 265(1390):33-38.

Loreau, M. and Ebenhöh, W. (1994). Competitive exclusion and coexistence of species with complex life cycles. Theoretical Population Biology, 46(1):58-77.

Lotka, A. J. (1925). The empirical element in population forecasts. Journal of the American Statistical Association, 20:569-570.

MacArthur, R. H. (1970). Species packing and competitive equilibrium for many species. Theoretical Population Biology, 1:1-11.

MacArthur, R. H. and Levins, R. (1964). Competition, habitat selection and character displacement in a patchy environment. Proceedings of the National Academy of Sciences of the United States of America, 51(6):1207-1210.

MacArthur, R. H. and Wilson, E. O. (1967). The Theory of Island Biogeography. 1035 Princeton University Press, Princeton, NJ.

Marler, R. T. and Arora, J. S. (2004). Survey of multi-objective optimization methods for engineering. Structural and Multidisciplinary Optimization, 26(6):369-395.

Massol, F. and Crochet, P.-A. (2008). Do animal personalities emerge? Nature, 451(7182):E8-E9.

Matsuda, H. and Abrams, P. A. (1994). Runaway evolution to self-extinction under asymmetrical competition. Evolution, 48(6):1764-1772. 
McGill, B. J., Enquist, B. J., Weiher, E., and Westoby, M. (2006). Rebuilding community ecology from functional traits. Trends in Ecology 83 Evolution, 21(4):178-185.

Meszéna, G., Gyllenberg, M., Pásztor, L., and Metz, J. A. J. (2006). Competitive exclusion and limiting similarity: a unified theory. Theoretical Population Biology, 69(1):68-87.

Meszéna, G. and Metz, J. A. J. (1999). Species diversity and population regulation: the importance of environmental feedback dimensionality. IIASA Working Paper WP-99-045.

Metz, J. A. J., Geritz, S. A. H., Meszéna, G., Jacobs, F. J. A., and van Heerwaarden, J. S. (1996). Adaptive dynamics, a geometrical study of the consequences of nearly faithful reproduction. In Stochastic and Spatial Structures of Dynamical Systems. Proceedings of the Royal Dutch Academy of Science, pages $183-231$.

Metz, J. A. J., Mylius, S. D., and Diekmann, O. (2008). When does evolution optimize? Evolutionary Ecology Research, 10:629-654.

Miller, T. E., Burns, J. H., Munguia, P., Walters, E. L., Kneitel, J. M., Richards, P. M., Mouquet, N., and Buckley, H. L. (2005). A critical review of twenty years' use of the resource-ratio theory. The American Naturalist, 165(4):43948.

Monod, J. (1950). Theory and application of the technique of continuous culture. Annals of the Pasteur Institute, 79:590-410.

1065 Murdoch, W. W. (1969). Switching in general predators: experiments on predator specificity and stability of prey populations. Ecological Monographs, $39(4): 335-354$. 
Murdoch, W. W., Avery, S., and Smyth, M. E. B. (1975). Switching in predatory fish. Ecology, 56(5):1094-1105.

Norberg, J. (2013). Biodiversity and ecosystem functioning: a complex adaptive systems approach. Limnology and Oceanography, 49(4):1269-1277.

Novick, A. and Szilard, L. (1950). Description of the chemostat. Science, 112:715-716.

Pareto, V. (1906). Manuale di Economia Politica con una Introduzione alla Scienza Sociale. Societa Editrice Libraria, Milano.

Parvinen, K. (2005). Evolutionary suicide. Acta Biotheoretica, 53(3):241-264.

Pietrewicz, A. T. and Kamil, A. C. (1979). Search image formation in the blue jay (Cyanocitta cristata). Science (New York, N.Y.), 204(4399):1332-1333.

Rankin, D. J. and López-Sepulcre, A. (2005). Can adaptation lead to extinction? Oikos, 111(3):616-619.

Rietkerk, M., Dekker, S. C., de Ruiter, P. C., and van de Koppel, J. (2004). Self-organized patchiness and catastrophic shifts in ecosystems. Science, 305(5692):1926-9.

Rueffler, C., Van Dooren, T. J. M., and Metz, J. A. J. (2004). Adaptive walks on changing landscapes: Levins' approach extended. Theoretical Population Biology, 65(2):165-178.

Ryabov, A. B. and Blasius, B. (2011). A graphical theory of competition on spatial resource gradients. Ecology Letters, 14(3):220-228.

Samuelson, P. A. (1947). Foundations of Economic Analysis. Harvard University Press, Cambridge. 
Sauterey, B., Ward, B. A., Follows, M. J., Bowler, C., and Claessen, D. (2015).

When everything is not everywhere but species evolve: an alternative method to model adaptive properties of marine ecosystems. Journal of Plankton Research, 37(1):28-47.

Schade, J. D., Espeleta, J. F., Klausmeier, C. A., McGroddy, M. E., Thomas, S. A., and Zhang, L. (2005). A conceptual framework for ecosystem stoichiometry: balancing resource supply and demand. Oikos, 109(1):40-51.

Scheffer, M., Carpenter, S., Foley, J. A., Folke, C., and Walker, B. (2001). Catastrophic shifts in ecosystems. Nature, 413:591-596. shifts result in niche partitioning between two consumer species irrespective of competitive abilities. The American Naturalist, 176(5):625-637.

Schreiber, S. J. and Rudolf, V. H. W. (2008). Crossing habitat boundaries: coupling dynamics of ecosystems through complex life cycles. Ecology Letters, $1105 \quad 11(6): 576-87$.

Schreiber, S. J. and Tobiason, G. A. (2003). The evolution of resource use. Journal of Mathematical Biology, 47:56-78.

Seppelt, R., Lautenbach, S., and Volk, M. (2013). Identifying trade-offs between ecosystem services, land use, and biodiversity: a plea for combining scenario analysis and optimization on different spatial scales. Current Opinion in Environmental Sustainability, 5(5):458-463.

Shoresh, N., Hegreness, M., and Kishony, R. (2008). Evolution exacerbates the paradox of the plankton. Proceedings of the National Academy of Sciences of the United States of America, 105(34):12365-12369. 
Smith, S. L., Pahlow, M., Merico, A., and Wirtz, K. W. (2011). Optimalitybased modeling of planktonic organisms. Limnology and Oceanography, 56(6):2080-2094.

Strogatz, S. H. (2015). Nonlinear Dynamics and Chaos: With Applications to Physics, Biology, Chemistry, and Engineering. Westview Press, Boulder, CO.

${ }_{1120}$ Stuart, Y. E., Campbell, T. S., Hohenlohe, P. A., Reynolds, R. G., Revell, L. J., and Losos, J. B. (2014). Rapid evolution of a native species following invasion by a congener. Science, 346(6208):463-466.

Szilágyi, A. and Meszéna, G. (2009). Limiting similarity and niche theory for structured populations. Journal of Theoretical Biology, 258:27-37.

${ }_{1125}$ Thomas, M. K., Kremer, C. T., Klausmeier, C. A., and Litchman, E. (2012). A global pattern of thermal adaptation in marine phytoplankton. Science, 338:1085-1089.

Thompson, J. N. (1998). Rapid evolution as an ecological process. Trends in Ecology \&3 Evolution, 13(8):329-32.

1130 Tilman, D. (1980). Resources: a graphical-mechanistic approach to competition and predation. The American Naturalist, 116(3):362-393.

Tilman, D. (1982). Resource Competition and Community Structure. Princeton University Press, Princeton, NJ.

Tilman, D. (1988). Plant Strategies and the Dynamics and Structure of Plant 1135 Communities. Princeton University Press, Princeton, NJ.

Volterra, V. (1926). Fluctuations in the abundance of a species considered mathematically. Nature, 118(2972):558-560. 
Westoby, M. and Wright, I. J. (2006). Land-plant ecology on the basis of functional traits. Trends in Ecology 83 Evolution, 21(5):261-268.

${ }_{1140}$ Wolf, M., van Doorn, G. S., Leimar, O., and Weissing, F. J. (2007). Life-history trade-offs favour the evolution of animal personalities. Nature, 447(7144):581584 .

Wolf, M., van Doorn, G. S., Leimar, O., and Weissing, F. J. (2008). Wolf et al. reply. Nature, 451(7182):E9-E10.

1145 Wolkowicz, G. S. K. and Lu, Z. (1992). Global dynamics of a mathematical model of competition in the chemostat: general response functions and differential death rates. SIAM Journal on Applied Mathematics, 52(1):222-233.

Yoshida, T., Jones, L. E., Ellner, S. P., Fussmann, G. F., and Hairston, N. G. (2003). Rapid evolution drives ecological dynamics in a predator-prey system. $1150 \quad$ Nature, 424(6946):303-306.

Zu, J., Yuan, B., and Du, J. (2015). Top predators induce the evolutionary diversification of intermediate predator species. Journal of Theoretical Biology, $387: 1-12$.

\section{A. Transformation toward decoupled chemostat dynamics}

1155 We provide here two examples of change of variables that enable one to map more general regulating factors dynamics to a chemostat dynamics form presented in equations (1b). This allows one to apply the graphical method presented in this paper to those extended situations after the change of variables.

\section{A.1. Logistic growth}

1160

First, let us consider that a regulating factor $R$ follows a logistic resource dynamics:

$$
\frac{d R}{d t}=r R\left(1-\frac{R}{K}\right)+\sum_{j=1}^{n} I_{j}(R) N_{j}
$$


Introducing the change of variables $\rho=1 / R$, it is straightforward that:

$$
\frac{d \rho}{d t}=r(\kappa-\rho)+\sum_{j=1}^{n} \chi_{j}(\rho) N_{j}
$$

1165 logistic growth in $R$ toward a chemostat dynamics in $\rho$. Note that the consumerresource relationship with the $N_{j}$ s is reversed by the change of variable: if a $N_{j}$ was consuming $R$, it is now feeding $\rho$. This can be understood by looking at the dimensions of the new variable: if $R$ is in individual per surface area, $\rho$ is 1170 conversely increases available surface area per individual.

\section{A.2. Linear coupling through diffusion}

Our second example considers two diffusion-coupled chemostats, thus following the intrinsic dynamics:

$$
\begin{aligned}
& \frac{d R_{1}}{d t}=l_{1}\left(S_{1}-R_{1}\right)-d_{12} R_{1}+d_{21} R_{2} \\
& \frac{d R_{2}}{d t}=l_{2}\left(S_{2}-R_{2}\right)+d_{12} R_{1}-d_{21} R_{2}
\end{aligned}
$$

1175 This system being linear, it can be rewritten under the general matrix form in the presence of interacting populations:

$$
\frac{d \boldsymbol{R}}{d t}=\boldsymbol{T}-\boldsymbol{M} \boldsymbol{R}+\sum_{j=1}^{n} \boldsymbol{I}_{j}(\boldsymbol{R}) N_{j}
$$

with

$$
\boldsymbol{T}=\left(\begin{array}{c}
l_{1} S_{1} \\
l_{2} S_{2}
\end{array}\right) \quad \text { and } \quad \boldsymbol{M}=\left(\begin{array}{cc}
l_{1}-d_{12} & d_{21} \\
d_{12} & l_{1}-d_{21}
\end{array}\right)
$$

Then, diagonalization gives $\boldsymbol{M}=\boldsymbol{P} \boldsymbol{D} \boldsymbol{P}^{-\mathbf{1}}$ with $\boldsymbol{D}=\operatorname{diag}\left(\lambda_{1}, \lambda_{2}\right)$ leading to:

$$
\frac{d \boldsymbol{\rho}}{d t}=\boldsymbol{D}(\boldsymbol{\sigma}-\boldsymbol{\rho})+\sum_{j=1}^{n} \chi_{j}(\boldsymbol{\rho}) N_{j}
$$


with $\boldsymbol{\rho}=\boldsymbol{P}^{-1} \boldsymbol{R}, \boldsymbol{\sigma}=\boldsymbol{D}^{-1} \boldsymbol{P}^{-1} \boldsymbol{T}$ and $\boldsymbol{\chi}_{j}(\boldsymbol{\rho})=\boldsymbol{P}^{-1} \boldsymbol{I}_{j}(\boldsymbol{P} \boldsymbol{\rho})$. As $\boldsymbol{D}$ is a diagonal matrix, the regulating factor vector $\boldsymbol{\rho}$ nows follows decoupled chemostat dynamics.

\section{B. Analytical study of ecological equilibria for the two consumers on two resources system}

\section{B.1. Model}

In the case of two consumers competing for two resource in a chemostat, model (1) can be rewritten:

$$
\begin{aligned}
\frac{d N_{i}}{d t} & =w_{i}\left(R_{1}, R_{2}\right) N_{i} \\
\frac{d R_{i}}{d t} & =l_{i}\left(S_{i}-R_{i}\right)+\sum_{j=1}^{2} I_{i j}\left(R_{1}, R_{2}\right) N_{j}
\end{aligned}
$$

Let us first classify the different equilibria of the system and characterize their local stability.

\section{B.2. Equilibria}

Those equations present different kinds of solutions at equilibrium:

Equilibrium (0). corresponds to the case where both populations are absent, i.e. $N_{1}=N_{2}=0$. Then $R_{1}=S_{1}$ and $R_{2}=S_{2}$.

Equilibrium (1). corresponds to the case where only population 2 is absent, i.e. $N_{1} \neq 0$ and $N_{2}=0$. The system can be rewritten:

$$
\begin{aligned}
0 & =w_{1}\left(R_{1}, R_{2}\right) \\
\tilde{I}_{12}\left(R_{1}, R_{2}\right)\left(S_{1}-R_{1}\right) & =\tilde{I}_{11}\left(R_{1}, R_{2}\right)\left(S_{2}-R_{2}\right) \\
N_{1} & =\frac{\left(R_{1}-S_{1}\right)}{\tilde{I}_{11}\left(R_{1}, R_{2}\right)}
\end{aligned}
$$

where we have used the simplifying notation $\tilde{I}_{i j}=I_{i j} / l_{i}$. The regulating factor values at equilibrium $\left(R_{1}, R_{2}\right)$ are obtained by solving the first two equations together. $N_{1}$ is then deduced from the result using the third equation. 
Equilibrium (2). corresponds to the case where only population 1 is absent, i.e. $N_{2} \neq 0$ and $N_{1}=0$. The equilibrium values can be deduced from the previous paragraph by switching subscripts.

Equilibrium $(1+2)$. corresponds to the case where the two populations coexist, i.e. $N_{2} \neq 0$ and $N_{1} \neq 0$. Then $\left(R_{1}, R_{2}\right)$ are given after solving:

$$
\begin{aligned}
& 0=w_{1}\left(R_{1}, R_{2}\right) \\
& 0=w_{2}\left(R_{1}, R_{2}\right)
\end{aligned}
$$

The densities values at equilibrium follow with:

$$
\boldsymbol{N}=\tilde{\boldsymbol{I}}^{-1}(\boldsymbol{S}-\boldsymbol{R})
$$

where $\boldsymbol{N}=\left(N_{1}, N_{2}\right)^{T}, \boldsymbol{S}=\left(S_{1}, S_{2}\right)^{T}, \boldsymbol{R}=\left(R_{1}, R_{2}\right)^{T}$ and $\tilde{\boldsymbol{I}}$ is a 2 by 2 matrix with coefficients $\tilde{I}_{i j}=I_{i j} / l_{i}$. Note that $\tilde{\boldsymbol{I}}$ is invertible if and only if the 1210 renormalized impacts vectors of the two populations are not colinear, which is improbable in the absence of fine-tuning.

\section{B.3. Stability}

The stability of those different types of equilibria can be assessed introducing the Jacobian of the system:

$$
\boldsymbol{J}\left(N_{1}, N_{2}, R_{1}, R_{2}\right)=\left(\begin{array}{cccc}
w_{1} & 0 & \partial_{1} w_{1} N_{1} & \partial_{2} w_{1} N_{1} \\
0 & w_{2} & \partial_{1} w_{2} N_{2} & \partial_{2} w_{2} N_{2} \\
I_{11} & I_{12} & -l_{1}+\sum_{j=1}^{2} \partial_{1} I_{1 j} N_{j} & \sum_{j=1}^{2} \partial_{2} I_{1 j} N_{j} \\
I_{21} & I_{22} & \sum_{j=1}^{2} \partial_{1} I_{2 j} N_{j} & -l_{2}+\sum_{j=1}^{2} \partial_{2} I_{2 j} N_{j}
\end{array}\right)
$$

where we have omitted the explicit dependencies in $\left(R_{1}, R_{2}\right)$ and the notation $\partial_{i}$ stands for $\partial / \partial R_{i}$. This Jacobian can be evaluated for the different kinds of 
equilibria we have identified. It is not to be confused with the Jacobian of the fitness gradient $J$ of eq. (8).

1220 Equilibrium (0). The Jacobian can be rewritten as:

$$
\boldsymbol{J}=\left(\begin{array}{cccc}
w_{1} & 0 & 0 & 0 \\
0 & w_{2} & 0 & 0 \\
I_{11} & I_{12} & -l_{1} & 0 \\
I_{21} & I_{22} & 0 & -l_{2}
\end{array}\right)
$$

As $\left(R_{1}, R_{2}\right)=\left(S_{1}, S_{2}\right)$, the empty equilibrium is stable if both $w_{1}\left(S_{1}, S_{2}\right)<0$ and $w_{2}\left(S_{1}, S_{2}\right)<0$, which means that none of the two populations can invade. There is no other constraint as the chemostat dynamics are 'intrinsically' stable. Equilibrium (1). After permutation, the Jacobian can be rewritten as a blockdiagonal matrix:

$$
\boldsymbol{J}=\left(\begin{array}{cc}
w_{2} & \mathbf{0} \\
\mathbf{0} & \boldsymbol{K}
\end{array}\right)
$$

with

$$
\boldsymbol{K}=\left(\begin{array}{ccc}
0 & \partial_{1} w_{1} N_{1} & \partial_{2} w_{1} N_{1} \\
I_{11} & -l_{1}+\partial_{1} I_{11} N_{1} & \partial_{2} I_{11} N_{1} \\
I_{21} & \partial_{1} I_{21} N_{1} & -l_{2}+\partial_{2} I_{21} N_{1}
\end{array}\right)
$$

Thus, a first necessary condition is non-invasibility by population 2 through $w_{2}\left(R_{1}, R_{2}\right)<0$. Routh-Hurwitz criteria applied on $\boldsymbol{K}$ gives a second necessary condition $\operatorname{det} \boldsymbol{K}<0$. It is actually possible to show that:

$$
\operatorname{det} \boldsymbol{K}=l_{2} N_{1} I_{11} \frac{\partial w_{1}}{\partial R_{1}} / \frac{\partial R_{2}}{\partial S_{2}}
$$

The object $\partial R_{2} / \partial S_{2}$ has an intuitive geometrical interpretation linked to the 1235 envelope of the impact rays. The scheme is similar to the one developed in the main text in the eco-evolutionary context (see supply point map in section 2.3). Indeed, the family of impact rays associated with a given ZNGI can possess an 
envelope. When it is the case, a given impact ray is tangent to its envelope at a particular point. The line portion of the impact ray situated between its origin 1240 and this point corresponds to supply points satisfying $\partial R_{1} /\left.\partial s_{1}\right|_{Z}>0$ while the other part corresponds to $\partial R_{1} /\left.\partial s_{1}\right|_{Z}<0$. In the classical consumer-resource situation where $I_{11} \partial w_{1} / \partial R_{1}<0$, this means that only the supply points situated before the envelope on the impact ray map to stable equilibria, the other ones being unstable. This graphical criterion shares strong similarities with

1245 the eco-evolutionary case presented in the main text. Note that the condition $\operatorname{det} \boldsymbol{K}<0$ is necessary but not sufficient to ensure stability.

Equilibrium (2). can be deduced from the previous paragraph by switching subscripts. Note that we get the necessary condition $\operatorname{det} \boldsymbol{K}^{\prime}<0$ for stability with :

$$
\operatorname{det} \boldsymbol{K}^{\prime}=l_{1} N_{2} I_{22} \frac{\partial w_{2}}{\partial R_{2}} / \frac{\partial R_{1}}{\partial S_{1}}
$$

Equilibrium $(1+2)$. The Jacobian can be rewritten as:

$$
\boldsymbol{J}=\left(\begin{array}{cccc}
0 & 0 & \partial_{1} w_{1} N_{1} & \partial_{2} w_{1} N_{1} \\
0 & 0 & \partial_{1} w_{2} N_{2} & \partial_{2} w_{2} N_{2} \\
I_{11} & I_{12} & -l_{1}+\sum_{j=1}^{2} \partial_{1} I_{1 j} N_{j} & \sum_{j=1}^{2} \partial_{2} I_{1 j} N_{j} \\
I_{21} & I_{22} & \sum_{j=1}^{2} \partial_{1} I_{2 j} N_{j} & -l_{2}+\sum_{j=1}^{2} \partial_{2} I_{2 j} N_{j}
\end{array}\right)
$$

The necessary condition for stability $\operatorname{det} \boldsymbol{J}>0$ can be obtained where:

$$
\operatorname{det} \boldsymbol{J}=\left(I_{11} I_{22}-I_{12} I_{21}\right)\left(\frac{\partial w_{1}}{\partial R_{1}} \frac{\partial w_{2}}{\partial R_{2}}-\frac{\partial w_{1}}{\partial R_{2}} \frac{\partial w_{2}}{\partial R_{1}}\right) N_{1} N_{2}
$$

$1 \mathbf{2 5 5}$ this is the mutual invasibility criterion of eq. (3) in the main text. We recognize in eq. (B.12) the general decomposition of $\operatorname{det} \boldsymbol{J}$ as the product of the impact and sensitivity map volumes (Meszéna et al., 2006). 


\section{Demonstration of the geometrical relationships in the $k$-dimensional traitspace case}

The aim of this section is to link the ZNGI and impact ray envelope properties to the eco-evolutionary properties of the corresponding singular points. We restrict our attention to the case of two regulating factors $R_{1}$ and $R_{2}$, for a completely general $k$-dimensional trait $\boldsymbol{x}$.

\section{C.1. Monomorphic singular point}

When there is only one singular population in the system, the eco-evolutionary invasion analysis of eq. (5) can be generalized to the $k$-dimensional case as:

$$
\begin{array}{r}
w(\boldsymbol{R}, \boldsymbol{x})=0 \\
\partial_{\boldsymbol{x}} w(\boldsymbol{R}, \boldsymbol{x})=\mathbf{0}
\end{array}
$$

where we have used the simplifying notation for the fitness gradient:

$$
\partial_{\boldsymbol{x}} w(\boldsymbol{R}, \boldsymbol{x}) \equiv\left[\frac{\partial w(\boldsymbol{R}(\boldsymbol{y}), \boldsymbol{x})}{\partial \boldsymbol{x}}\right]_{y=x}
$$

the notation $\partial / \partial_{\boldsymbol{x}}$ standing for a nabla operator along $\boldsymbol{x}$. Graphically, we recognized in the main text that this set of equations parametrizes the ZNGI envelope. This particular expression of the fitness gradient as a partial derivative along its second coordinate is specific to the fact that mutant and resident only interact indirectly through the regulating factors. This has to be combined with the supply point map:

$$
v(\boldsymbol{S}, \boldsymbol{R}, \boldsymbol{x})=0
$$

with $v(\boldsymbol{S}, \boldsymbol{R}, \boldsymbol{x})=\left(S_{1}-R_{1}\right) \tilde{I}_{2}(\boldsymbol{R}, \boldsymbol{x})-\left(S_{2}-R_{2}\right) \tilde{I}_{1}(\boldsymbol{R}, \boldsymbol{x})$. Jointly solving this system gives the singular point trait values.

How can we link the tangent ZNGI and ZNGI envelope relative curvature to the properties of its corresponding singular points? As was done in the main 
text in the unidimensional case, we need to introduce the Hessian matrix of the invasion fitness $\boldsymbol{H}$ :

$$
\boldsymbol{H}(\boldsymbol{x})=\left[\frac{\partial}{\partial \boldsymbol{x}} \frac{\partial}{\partial \boldsymbol{x}^{T}} w(\boldsymbol{R}(\boldsymbol{y}), \boldsymbol{x})\right]_{y=x} \equiv \partial_{\boldsymbol{x}} \partial_{\boldsymbol{x}}^{T} w
$$

The ZNGI and envelope curvatures at the singular point are both given by second derivatives. The ZNGI curvature can be obtained differentiating $w\left(R_{1}, R_{2}, \boldsymbol{x}\right)=$ 0 twice with respect to $R_{1}$, where $R_{2}=f\left(R_{1}\right)$ :

$$
\begin{aligned}
& -\left.\partial_{R_{2}} w \cdot \frac{\partial R_{2}}{\partial R_{1}}\right|_{Z}=\partial_{R_{1}} w \\
& -\left.\partial_{R_{2}} w \cdot \frac{\partial^{2} R_{2}}{\partial R_{1}^{2}}\right|_{Z}=\partial_{R_{1}}^{2} w+2 \frac{\partial R_{2}}{\partial R_{1}} \partial_{R_{2}} \partial_{R_{1}} w+\left(\frac{\partial R_{2}}{\partial R_{1}}\right)^{2} \partial_{R_{2}}^{2} w
\end{aligned}
$$

where $\boldsymbol{x}$ has been kept fixed for this calculation as we are interested by the properties of the tangent ZNGI. This is not the case for the ZNGI envelope. The envelope curvature is obtained differentiating $w\left(R_{1}, R_{2}, \boldsymbol{x}\right)=0$ twice with respect to $R_{1}$ and using $\partial_{\boldsymbol{x}} w\left(R_{1}, R_{2}, \boldsymbol{x}\right)=\mathbf{0}$, where $R_{2}=g\left(R_{1}\right)$ and $\boldsymbol{x}=\boldsymbol{h}\left(R_{1}\right)$ :

$$
\begin{aligned}
-\left.\partial_{R_{2}} w \cdot \frac{\partial R_{2}}{\partial R_{1}}\right|_{\mathrm{E}} & =\partial_{R_{1}} w \\
-\left.\partial_{R_{2}} w \cdot \frac{\partial^{2} R_{2}}{\partial R_{1}^{2}}\right|_{\mathrm{E}} & =\partial_{R_{1}}^{2} w+2 \frac{\partial R_{2}}{\partial R_{1}} \partial_{R_{2}} \partial_{R_{1}} w+\left(\frac{\partial R_{2}}{\partial R_{1}}\right)^{2} \partial_{R_{2}}^{2} w \\
& +\frac{\partial \boldsymbol{x}^{T}}{\partial R_{1}}\left(\partial_{R_{1}} \partial_{\boldsymbol{x}} w+\frac{\partial R_{2}}{\partial R_{1}} \partial_{R_{2}} \partial_{\boldsymbol{x}} w\right)
\end{aligned}
$$

Note that the first order derivatives are the same for the ZNGI and the envelope, which is a result of their tangency and is known in economics as the "Envelope Theorem" (Samuelson, 1947). However, the second derivatives differ by a term that accounts for the fact that $\boldsymbol{x}$ also varies along the envelope. Differentiating

${ }_{1295} \partial_{\boldsymbol{x}} w\left(R_{1}, R_{2}, \boldsymbol{x}\right)=\mathbf{0}$ once with respect to $R_{1}$ shows how the last term of (C.9) is actually related to the Hessian:

$$
\frac{\partial \boldsymbol{x}^{T}}{\partial R_{1}}\left(\partial_{R_{1}} \partial_{\boldsymbol{x}} w+\frac{\partial R_{2}}{\partial R_{1}} \partial_{R_{2}} \partial_{\boldsymbol{x}} w\right)=-\frac{\partial \boldsymbol{x}^{T}}{\partial R_{1}} \boldsymbol{H} \frac{\partial \boldsymbol{x}}{\partial R_{1}}
$$


In conclusion, combining all the previous results as the sum $-($ C.9 $)+($ C.7 $)-($ C.10) and using the fact that $\partial R_{2} / \partial R_{1}$ coincides for both ZNGI and envelope leads to the final result:

1300

$$
\frac{\partial w}{\partial R_{2}}\left(\left.\frac{\partial^{2} R_{2}}{\partial R_{1}^{2}}\right|_{\mathrm{E}}-\left.\frac{\partial^{2} R_{2}}{\partial R_{1}^{2}}\right|_{\mathrm{Z}}\right)=\frac{d \boldsymbol{x}^{T}}{d R_{1}} \cdot \boldsymbol{H} \cdot \frac{d \boldsymbol{x}}{d R_{1}}
$$

When $\boldsymbol{x}$ is a scalar, the latter expression directly rewrites as eq. (7), which concludes the proof.

The second result presented in the main text relates the convergence properties of a singular point to the impact ray envelope. Let us introduce the Jacobian of the fitness gradient $\boldsymbol{J}$ :

$$
\begin{aligned}
\boldsymbol{J}(\boldsymbol{x}) & =\frac{\partial}{\partial \boldsymbol{x}}\left[\frac{\partial}{\partial \boldsymbol{x}^{T}} w(\boldsymbol{R}(\boldsymbol{y}), \boldsymbol{x})\right]_{y=x} \\
& \equiv\left(\partial_{\boldsymbol{x}}+\partial_{\boldsymbol{x}} \boldsymbol{R}^{T} . \partial_{\boldsymbol{R}}\right) \partial_{\boldsymbol{x}}^{T} w
\end{aligned}
$$

We thus have:

$$
\boldsymbol{J}(\boldsymbol{x})=\boldsymbol{H}(\boldsymbol{x})+\partial_{\boldsymbol{x}} \boldsymbol{R}^{T} . \partial_{\boldsymbol{R}} \partial_{\boldsymbol{x}}^{T} w
$$

It is very important to understand that the dependency of $\boldsymbol{R}$ in $\boldsymbol{x}$ depicted by ${ }_{1310}$ the term $\partial_{\boldsymbol{x}} \boldsymbol{R}^{T}$ comes from solving completely the ecological system by combining ZNGI and impact ray equations (C.1,C.4) for $\boldsymbol{S}$ fixed. This has to be done for any strategy, singular or not. Note that this object is not directly related to the expression $d \boldsymbol{x} / d R_{1}$ manipulated above, which tracks how a singular strategy varies along the envelope. Differentiating $w(\boldsymbol{R}, \boldsymbol{x})=0$ and $v(\boldsymbol{S}, \boldsymbol{R}, \boldsymbol{x})=0$ with 1315 respect to $\boldsymbol{x}$ and evaluating it at a singular point gives the following relation- 
ships:

$$
\begin{aligned}
& \partial_{\boldsymbol{x}} R_{1}=-\partial_{\boldsymbol{x}} v /\left(\partial_{R_{1}} v+\frac{\partial R_{2}}{\partial R_{1}} \partial_{R_{2}} v\right) \\
& \partial_{\boldsymbol{x}} R_{2}=\frac{\partial R_{2}}{\partial R_{1}} \partial_{\boldsymbol{x}} R_{1}
\end{aligned}
$$

Let us first use eq. (C.16) only to rewrite:

$$
\boldsymbol{J}-\boldsymbol{H}=\partial_{\boldsymbol{x}} R_{1}\left(\partial_{R_{1}} \partial_{\boldsymbol{x}}^{T} w+\frac{\partial R_{2}}{\partial R_{1}} \partial_{R_{2}} \partial_{\boldsymbol{x}}^{T} w\right)
$$

after multiplying eq. (C.17) on the right by $d \boldsymbol{x} / d R_{1}$, the RHS reads as the transpose of the LHS of eq. (C.10). When also multiplied by $d \boldsymbol{x}^{T} / d R_{1}$ on the left, it leads to:

$$
\frac{d \boldsymbol{x}^{T}}{d R_{1}} \boldsymbol{J} \frac{d \boldsymbol{x}}{d R_{1}}=\left(1-\frac{d \boldsymbol{x}^{T}}{d R_{1}} \cdot \partial_{\boldsymbol{x}} R_{1}\right) \frac{d \boldsymbol{x}^{T}}{d R_{1}} \boldsymbol{H} \frac{d \boldsymbol{x}}{d R_{1}}
$$

Finally, coming back to the expression of $\partial_{\boldsymbol{x}} R_{1}$ given by eq. (C.16):

$$
\begin{aligned}
1-\frac{d \boldsymbol{x}^{T}}{d R_{1}} . \partial_{\boldsymbol{x}} R_{1} & =\left(\partial_{R_{1}} v+\frac{\partial R_{2}}{\partial R_{1}} \partial_{R_{2}} v+\frac{d \boldsymbol{x}^{T}}{d R_{1}} \cdot \partial_{\boldsymbol{x}} v\right) /\left(\partial_{R_{1}} v+\frac{\partial R_{2}}{\partial R_{1}} \partial_{R_{2}} v\right) \\
& \left.\equiv \frac{d v}{d R_{1}}\right|_{\mathrm{E}} /\left.\frac{d v}{d R_{1}}\right|_{\mathrm{Z}}
\end{aligned}
$$

Now, there is a last step to make the link with the supply point map. Differentiating (C.4) once with respect to $S_{1}$, along a ZNGI and the envelope respectively leads to:

$$
\begin{aligned}
& \left.\frac{\partial R_{1}}{\partial S_{1}}\right|_{\mathrm{Z}}=-\tilde{I}_{2} /\left.\frac{d v}{d R_{1}}\right|_{\mathrm{Z}} \\
& \left.\frac{\partial R_{1}}{\partial S_{1}}\right|_{\mathrm{E}}=-\tilde{I}_{2} /\left.\frac{d v}{d R_{1}}\right|_{\mathrm{E}}
\end{aligned}
$$

Putting the pieces together, we finally get the result:

$$
\left.\frac{\partial R_{1}}{\partial s_{1}}\right|_{\mathrm{E}} \frac{d \boldsymbol{x}^{T}}{d R_{1}} \boldsymbol{J} \frac{d \boldsymbol{x}}{d R_{1}}=\left.\frac{\partial R_{1}}{\partial s_{1}}\right|_{\mathrm{Z}} \frac{d \boldsymbol{x}^{T}}{d R_{1}} \boldsymbol{H} \frac{d \boldsymbol{x}}{d R_{1}}
$$


When $\boldsymbol{x}$ is a scalar and $d x / d R_{1} \neq 0$, the latter expression directly rewrites as 1330 eq. (9), which concludes the proof.

Using the ecological stability criteria (B.10) obtained in the previous section for a single-population, we have:

$$
\left.\frac{\partial R_{1}}{\partial s_{1}}\right|_{\mathrm{Z}}=-\beta \tilde{I}_{2} \frac{\partial w}{\partial R_{2}}
$$

${ }^{1335}$ where $\beta$ is a positive function when the ecological equilibrium is stable and the two other terms of the RHS are related to the relationship between the population and the regulating factor $R_{2}$. For the usual consumer-resource and predator-prey interactions, this thus leads to $\partial R_{1} / \partial s_{1}>0$.

${ }_{1340}$ Note that the two main relationships obtained here in the case of a general $k$-dimensional trait $\boldsymbol{x}$ only give information on the Hessian and Jacobian of the eco-evolutionary system along the envelope, i.e. the direction $d \boldsymbol{x} / d R_{1}$. In the one dimensional case, this is not a problem as soon as this direction exists (is non-zero): it leads to a squared term with thus no effect of its sign for the first ${ }_{1345}$ result or can be simplified in the second result. For dimensions greater than one, this projection leads to insufficient information on the multiple eigenvalues of both the Hessian and Jacobian matrices, not allowing to conclude on the ESS and convergent properties of the singular points based only on the relative position between the ZNGI and its envelope. Also note that contrary to the one 1350 dimensional case, eco-evolutionary stability in the $k$-dimensional case may also depend on the specific shape of the mutation process (Leimar, 2009). 


\section{C.2. Dimorphic singular point}

When there are two coexisting singular populations in the system with traits $\boldsymbol{x}_{\mathbf{1}} \neq \boldsymbol{x}_{\mathbf{2}}$, the eco-evolutionary invasion analysis reads:

$$
\begin{array}{r}
w\left(\boldsymbol{R}, \boldsymbol{x}_{\boldsymbol{i}}\right)=0 \\
\partial_{\boldsymbol{x}} w\left(\boldsymbol{R}, \boldsymbol{x}_{\boldsymbol{i}}\right)=\mathbf{0}
\end{array}
$$

1355

with $i=1,2$. As in the ecological case, this is enough to fully determine the regulating factors at the eco-evolutionary equilibrium, thus the supply point map is not needed here. According to the adaptive dynamics picture, the ecoevolutionary properties of this singular coalition directly emerges from those of its constituents. Thus, we still have:

$$
\frac{\partial w}{\partial R_{2}}\left(\left.\frac{\partial^{2} R_{2}}{\partial R_{1}^{2}}\right|_{\mathrm{E}}-\left.\frac{\partial^{2} R_{2}}{\partial R_{1}^{2}}\right|_{\mathrm{Z}}\right)=\frac{d \boldsymbol{x}_{\boldsymbol{i}}^{T}}{d R_{1}} \cdot \boldsymbol{H} \cdot \frac{d \boldsymbol{x}_{\boldsymbol{i}}}{d R_{1}}
$$

and thus this coalition is evolutionarily stable if and only if both coexisting strategies satisfy the geometrical condition relatively to their local envelope. The situation is a bit different for the convergence characteristics as $\boldsymbol{R}$ is obtained without the supply point map (and is thus independent of it) in the dimorphic case. Differentiating $w\left(\boldsymbol{R}, \boldsymbol{x}_{\mathbf{1}}\right)=0$ and $w\left(\boldsymbol{R}, \boldsymbol{x}_{\mathbf{2}}\right)=0$ with respect to $\boldsymbol{x}_{\boldsymbol{i}}$ and evaluating it at the singular point where $R_{2}=g\left(\boldsymbol{x}_{\mathbf{1}}, \boldsymbol{x}_{\mathbf{2}}\right)$ gives the trivial result:

$$
\partial_{\boldsymbol{x}_{\boldsymbol{i}}} R_{1}=\partial_{\boldsymbol{x}_{\boldsymbol{i}}} R_{2}=\mathbf{0}
$$

Thus, the regulating factors at equilibrium around a singular coalition are linearly independent from the traits of this coalition. As a result, $\boldsymbol{J}=\boldsymbol{H}$ for each of the two coexisting strategies, so the convergence properties are automatically linked to the invasion one. This means that ESS coalitions are automatically CSS ones and further branching is excluded. This result obtained here in the case of a satured dimorphism (two populations on two regulating factors) 
strongly echoes to the situation of a single population evolving on a single re1375 source, and can be generalized to any number of regulating factors (Kisdi and Geritz, 2016). 